\title{
Dynamical correlations in the electron gas
}

\author{
K. Sturm and A. Gusarov* \\ Institut für Festkörperforschung, Forschungszentrum Jülich GmbH, D-52425 Jülich, Germany
}

(Received 8 May 2000)

\begin{abstract}
Dynamical correlations in the homogeneous electron gas are a central issue in time-dependent density functional theory and are investigated here by evaluating exactly the leading corrections to the RPA proper polarizability. We obtain $\operatorname{Im} \epsilon(k, \omega)$ at arbitrary $k$ and $\omega$ outside the (single) particle-hole excitation spectrum. We calculate the imaginary part of the local field factor $\operatorname{Im} G(k, \omega)$ and compare $\operatorname{Im} \epsilon(k, \omega)$ with contributions arising from band structure effects in Al. These results are applied to Al to discuss their relative importance on plasmon damping and on the high frequency tail in the dynamical structure factor. Dynamical correlations mediated by the crystal potential contribute to the optical absorption of alkali metals below the onset of interband transitions.
\end{abstract}

\section{INTRODUCTION}

\section{A. Motivation}

Correlations in electron systems arise from the Coulomb interaction and from the Pauli principle and are essential to the development of approximations in density-functional theory (DFT). Dynamical correlations, in particular, are central to time-dependent density-functional theory (TDFT), a very active area of research. ${ }^{1}$ As in DFT, approximations for dynamical correlation effects are often based on the results for the homogeneous electron gas. Here we present exact results for dynamical correlations in the density response of the homogeneous electron gas in the high-density limit.

Before the advent of TDFT many approximations were proposed, ${ }^{2}$ beginning with the pioneering work of Hubbard. ${ }^{3}$ Exchange and correlation effects in the homogeneous electron gas beyond the random phase approximation (RPA) were accounted for by a local-field factor $G(k, \omega)$. Within a generalized mean-field approximation ${ }^{4}$ it is defined by

$$
\boldsymbol{\epsilon}(k, \omega)=1+v(k) \frac{\pi_{0}(k, \omega)}{1-v(k) G(k, \omega) \pi_{0}(k, \omega)} .
$$

Here $\pi_{0}(k, \omega)$ is the well-known independent particle response function and $v(k)=4 \pi e^{2} / k^{2}$. In most applications a frequency-independent local-field factor $G(k)$ has been used. The adiabatic local-density approximation is based on such a $G(k)$ in the limit $k \rightarrow 0$ and was applied to the calculation of the photoabsorption cross section by Zangwill and Soven. ${ }^{5}$ In an attempt to obtain a more realistic LDA for TDFT, Gross, Kohn, and Iwamoto (GKI) (Ref. 6) focused their attention on the time-dependent aspect. They designed a time-dependent local-density approximation (TLDA) from a complex frequency-dependent interpolation formula, with the exchange-correlation effects of the homogeneous electron gas defined by

$$
f_{x c}\left(\omega, n_{0}\right)=-\lim _{k \rightarrow 0} v(k) G(k, \omega) .
$$

$G(k, \omega)$ (in the small- $k$ limit) satisfies Kramers-Kronig relations, known sum rules, and a high-frequency limit, ${ }^{7} n_{0}$ is the ground-state density. It is then necessary to make a double local-density approximation for the inhomogeneous system. ${ }^{6}$ Though more refined than the adiabatic localdensity approximation, the double local approximations based on properties in the limit $k \rightarrow 0$ may still be questioned from a microscopic point of view. Furthermore, there is very little information on the microscopic mechanism of dynamic correlations.

Any attempt to answer these questions faces a complicated many-body problem. In principle, the leading corrections to the RPA can be determined by many-body perturbation theory in the high-density limit. One expands the proper polarizability in terms of irreducible diagrams. Böhm, Conti, and Tosi (BCT) (Ref. 8) pointed out in response to the GKI interpolation formula that an estimate of the complex $f_{x c}\left(\omega, n_{0}\right)$ could be obtained from the work of Hasegawa and Watabe $(\mathrm{HW})^{9}$ (see also DuBois and Kivelson ${ }^{10}$ ). HW evaluated the leading corrections to the RPA in the small- $k$ limit from the diagrams shown in Fig. 1. BCT observed considerable deviations from the GKI interpolation formula.
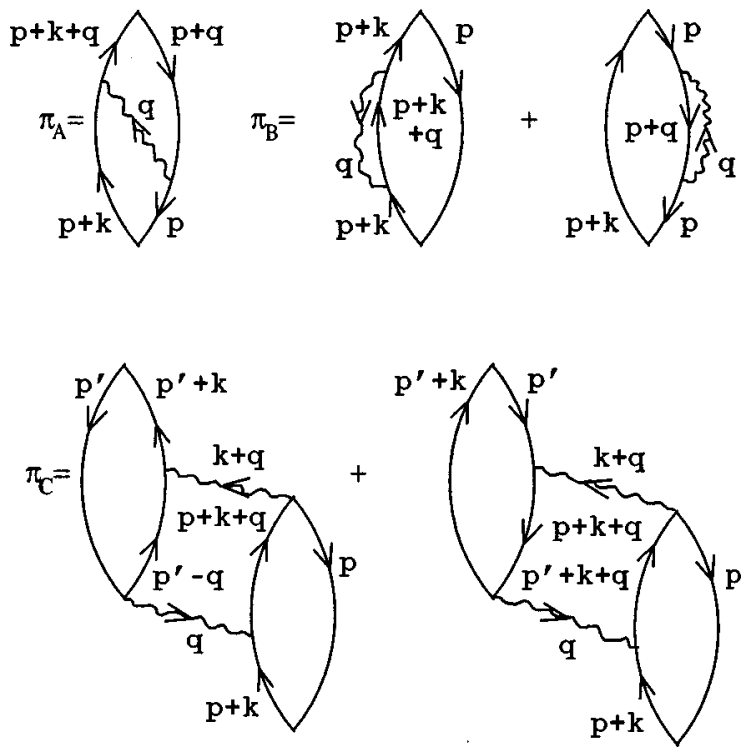

FIG. 1. The $\pi_{A}, \pi_{B}$, and $\pi_{C}$ diagrams are lowest order corrections to the RPA bubble. The wavy lines signify the dynamically screened Coulomb interaction. ( $p, p^{\prime}, q, k$ are four-dimensional vectors including frequencies.) 
To avoid the double local-density approximation in the GKI approach, it is important to extend the work of BCT to arbitrary $k$, because local, i.e., short-range correlations increase with increasing $k$. The evaluation of the diagrams in Fig. 1 for arbitrary $k$ and $\omega$ is therefore of general interest for the understanding of both static and time-dependent response properties. This was achieved recently for imaginary frequencies by Richardson and Ashcroft ${ }^{11}$ in order to determine the effective electron-electron interaction in the electron liquid. This is needed in many calculations, but an extension to real frequencies that can be tested directly by spectroscopic measurements is lacking. We have been able to reduce the numerical complications analytically to the extent that a numerical evaluation has become feasible.

The $\pi_{A}$ and $\pi_{B}$ diagrams of Fig. 1 are of first order in the dynamically RPA screened Coulomb interaction (wavy line). The $\pi_{C}$ diagrams of second order in the screened Coulomb interaction must be included for consistency, as first noticed by Perel' and Éliashberg ${ }^{12}$ and confirmed later by others. ${ }^{13-16}$ In particular, Geldart and Vosko (GV) (Ref. 14) demonstrated that a consistent set of diagrams generated in a self-consistent approximation (SCA) must include the $\pi_{C}$ diagrams. It will then give the correct $r_{s}$ dependence of the compressibility sum rule in the high-density limit, provided that the Coulomb interaction is screened dynamically.

Without screening, the $\pi_{A}$ and $\pi_{B}$ diagrams are the lowest-order Hartree-Fock diagrams that were evaluated many years ago. ${ }^{17}$ Although a substantial modification of the particle-hole (ph) excitations is observed, there are no excitations outside the ph spectrum. As a result, the first-order theory cannot explain well-known experimental observations such as the plasmon damping or the high-frequency tail of the dynamical structure factor $S(k, \omega)$, a defect that is overcome by dynamically screening the Coulomb interaction.

\section{B. Aim of this work}

The aim in the present work is to evaluate corrections to the RPA at arbitrary $(k, \omega)$ from the consistent set of diagrams shown in Fig. 1. We write the dielectric function $\epsilon(k, \omega)$ in terms of the proper polarizability $\pi_{p}(k, \omega)$, i.e.,

$$
\epsilon(k, \omega)=1+v(k) \pi_{p}(k, \omega) .
$$

$\pi_{p}(k, \omega)$ is expanded in terms of irreducible polarizability diagrams

$$
\begin{aligned}
\pi_{p}(k, \omega)= & \pi_{0}(k, \omega)+\pi_{A}(k, \omega)+\pi_{B}(k, \omega) \\
& +\pi_{C}(k, \omega)+\ldots
\end{aligned}
$$

Here $\pi_{p}(k, \omega)=\pi_{0}(k, \omega)$ defines the RPA, and the other terms in Eq. (1.4) defined by the diagrams in Fig. 1 represent the leading corrections to the RPA. We use the notation of Geldart and Vosko and adopt their sign convention for the polarizability.

Our calculation proceeds as follows. When standard methods $^{9,13}$ are applied to evaluate the diagrams one is left with seven-dimensional integrals, whose numerical evaluation is a formidable task that has prevented computation at arbitrary $k$ and $\omega$ until now. However, we have been able to reduce all expressions analytically to three-dimensional integrals, which are then evaluated numerically. Nevertheless, the evaluation of the diagrams is very complicated, and we restrict ourselves here to discussing $\operatorname{Im} \epsilon(k, \omega)$ outside the ph spectrum where the diagram $\pi_{0}(k, \omega)$ does not contribute. Thus

$$
\begin{aligned}
\operatorname{Im} \epsilon(k, \omega) & =v(k) \operatorname{Im}\left[\pi_{A}(k, \omega)+\pi_{B}(k, \omega)+\pi_{C}(k, \omega)\right] \\
& \equiv \operatorname{Im} \epsilon_{A B C}(k, \omega)
\end{aligned}
$$

In Sec. II the mathematical expressions corresponding to the diagrams of Fig. 1 are presented, and the details of the evaluation are outlined in the Appendix. The results of our numerical evaluation of the diagrams are discussed in Sec. III. For $\mathrm{Al}$ we also evaluate band structure effects for $\operatorname{Im} \epsilon(\mathbf{k}, \omega)$ and compare them with dynamical correlation effects. Consequences of the results on measurable physical effects are discussed in Sec. IV. Finally a summary and conclusions follow in Sec. V.

\section{MATHEMATICAL EXPRESSIONS OF THE DIAGRAMS IN FIG. 1}

Here we present the mathematical expressions of the diagrams of Fig. 1. For calculational convenience the diagrams are first defined along the imaginary frequency axis using temperature Green's functions (for details, see, for example, Ref. 13) by

$$
\begin{aligned}
\pi_{A}\left(k, \omega_{n}\right)= & \int \frac{d^{3} q}{(2 \pi)^{3}} \frac{1}{\beta} \sum_{m} U_{\mathbf{q}}\left(\alpha_{m}\right) \\
& \times 2 \int \frac{d^{3} p}{(2 \pi)^{3}} \frac{1}{\beta} \sum_{l} G_{\mathbf{p}}\left(\xi_{l}\right) G_{\mathbf{p}+\mathbf{k}}\left(\xi_{l}+\omega_{n}\right) \\
& \times G_{\mathbf{p}+\mathbf{q}}\left(\xi_{l}+\alpha_{m}\right) G_{\mathbf{p}+\mathbf{k}+\mathbf{q}}\left(\xi_{l}+\omega_{n}+\alpha_{m}\right),
\end{aligned}
$$

$$
\begin{aligned}
\pi_{B}\left(k, \omega_{n}\right)= & \int \frac{d^{3} q}{(2 \pi)^{3}} \frac{1}{\beta} \sum_{m} U_{\mathbf{q}}\left(\alpha_{m}\right) \\
& \times 2 \int \frac{d^{3} p}{(2 \pi)^{3}} \frac{1}{\beta} \sum_{l}\left\{\left[G_{\mathbf{p}}\left(\xi_{l}\right)\right]^{2} G_{\mathbf{p}+\mathbf{k}}\left(\xi_{l}+\omega_{n}\right)\right. \\
& \times G_{\mathbf{p}+\mathbf{q}}\left(\xi_{l}+\alpha_{m}\right)+\left[G_{\mathbf{p}+\mathbf{k}}\left(\xi_{l}+\omega_{n}\right)\right]^{2} \\
& \left.\times G_{\mathbf{p}}\left(\xi_{l}\right) G_{\mathbf{p}+\mathbf{k}+\mathbf{q}}\left(\xi_{l}+\omega_{n}+\alpha_{m}\right)\right\}
\end{aligned}
$$

and

$$
\begin{aligned}
\pi_{C}\left(k, \omega_{n}\right)= & \int \frac{d^{3} q}{(2 \pi)^{3}} \frac{1}{\beta} \sum_{m} U_{\mathbf{q}}\left(\alpha_{m}\right) U_{\mathbf{q}+\mathbf{k}}\left(\alpha_{m}+\omega_{n}\right) \\
& \times 2 \int \frac{d^{3} p}{(2 \pi)^{3}} 2 \int \frac{d^{3} p^{\prime}}{(2 \pi)^{3}} \frac{1}{\beta^{2}} \sum_{l, l^{\prime}} G_{\mathbf{p}^{\prime}}\left(\xi_{l^{\prime}}\right) \\
& \times G_{\mathbf{p}^{\prime}+\mathbf{k}}\left(\xi_{l^{\prime}}+\omega_{n}\right) G_{\mathbf{p}}\left(\xi_{l}\right) G_{\mathbf{p}+\mathbf{k}}\left(\xi_{l}+\omega_{n}\right) \\
& \times G_{\mathbf{p}+\mathbf{q}+\mathbf{k}}\left(\xi_{l}+\alpha_{m}+\omega_{n}\right) \\
& \times\left\{G_{\mathbf{p}^{\prime}+\mathbf{k}+\mathbf{q}}\left(\xi_{l^{\prime}}+\omega_{n}+\alpha_{m}\right)+G_{\mathbf{p}^{\prime}-\mathbf{q}}\left(\xi_{l^{\prime}}-\alpha_{m}\right)\right\} .
\end{aligned}
$$

Here $G_{\mathbf{P}}\left(\xi_{l}\right)$ is the Green's function for free Fermions

$$
G_{\mathbf{p}}\left(\xi_{l}\right)=\left(\xi_{l}-\varepsilon_{\mathbf{P}}\right)^{-1}
$$


with

$$
\xi_{l}=i \pi(2 l+1) / \beta+\varepsilon_{F} ; \quad l=0, \pm 1, \pm 2, \ldots
$$

and $\varepsilon_{\mathbf{p}}=\hbar^{2} p^{2} / 2 m_{e}, \quad m_{e}$ is the electron mass and $\beta$ $=\left(k_{B} T\right)^{-1} \cdot U_{\mathbf{q}}(z)$ is the dynamically RPA screened Coulomb potential at a complex frequency $z$

$$
U_{\mathbf{q}}(z)=v(\mathbf{q}) / \epsilon_{L}(q, z) .
$$

The imaginary frequencies $\alpha_{m}$ and $\omega_{n}$ are defined by

$$
\begin{gathered}
\alpha_{m}=i 2 \pi m / \beta ; \quad m=0, \pm 1, \pm 2, \ldots \\
\omega_{n}=i 2 \pi n / \beta ; \quad n=1,2,3, \ldots .
\end{gathered}
$$

The summations over $l$ and $l^{\prime}$ are performed by converting the sums into contour integrals. After some algebra we obtain

$$
\begin{aligned}
\pi_{A B}\left(k, \omega_{n}\right) \equiv & \pi_{A}\left(k, \omega_{n}\right)+\pi_{B}\left(k, \omega_{n}\right) \\
= & \int \frac{d^{3} q}{(2 \pi)^{3}} \frac{1}{\beta} \sum_{m} U_{\mathbf{q}}\left(\alpha_{m}\right) \\
& \times\left\{F_{1}\left(q, \alpha_{m} ; k, \omega_{n}\right)+F_{2}\left(q, \alpha_{m} ; k, \omega_{n}\right)\right\},
\end{aligned}
$$

and

$$
\begin{aligned}
\pi_{C}\left(k, \omega_{n}\right)= & \int \frac{d^{3} q}{(2 \pi)^{3}} \frac{1}{\beta} \sum_{m} U_{\mathbf{q}}\left(\alpha_{m}\right) U_{\mathbf{q}+\mathbf{k}}\left(\omega_{n}-\alpha_{m}\right) \\
& \times F_{3}\left(q,-\alpha_{m} ; k, \omega_{n}\right) \\
& \times\left[F_{3}\left(q,-\alpha_{m} ; k, \omega_{n}\right)+F_{3}\left(q, \alpha_{m} ; k,-\omega_{n}\right)\right],
\end{aligned}
$$

where

$$
\begin{aligned}
F_{1}\left(q, \alpha_{m} ; k, \omega_{n}\right) & \\
= & 2 \int \frac{d^{3} p}{(2 \pi)^{3}} f\left(\varepsilon_{\mathbf{p}}\right)\left[\frac{1}{\omega_{n}-\alpha_{m}+\varepsilon_{\mathbf{p}}-\varepsilon_{\mathbf{p}+\mathbf{k}+\mathbf{q}}}\right. \\
& \times\left(\frac{1}{\omega_{n}+\varepsilon_{\mathbf{p}}-\varepsilon_{\mathbf{p}+\mathbf{k}}}+\frac{1}{-\alpha_{m}+\varepsilon_{\mathbf{p}}-\varepsilon_{\mathbf{p}+\mathbf{q}}}\right)^{2} \\
& +\frac{1}{-\left(\omega_{n}-\alpha_{m}\right)+\varepsilon_{\mathbf{p}}-\varepsilon_{\mathbf{p}+\mathbf{k}+\mathbf{q}}} \\
& \left.\times\left(\frac{1}{-\omega_{n}+\varepsilon_{\mathbf{p}}-\varepsilon_{\mathbf{p}+\mathbf{k}}}+\frac{1}{\alpha_{m}+\varepsilon_{\mathbf{p}}-\varepsilon_{\mathbf{p}+\mathbf{q}}}\right)^{2}\right],
\end{aligned}
$$

$$
\begin{aligned}
& F_{2}\left(q, \alpha_{m} ; k, \omega_{n}\right) \\
& =2 \int \frac{d^{3} p}{(2 \pi)^{3}} \frac{\partial}{\partial \varepsilon_{\mathbf{p}}}\left[f\left(\varepsilon_{\mathbf{p}}\right) \frac{1}{\alpha_{m}+\varepsilon_{\mathbf{p}}-\varepsilon_{\mathbf{p}+\mathbf{q}}}\right. \\
& \left.\quad \times\left(\frac{1}{\omega_{n}+\varepsilon_{\mathbf{p}}-\varepsilon_{\mathbf{p}+\mathbf{k}}}+\frac{1}{-\omega_{n}+\varepsilon_{\mathbf{p}}-\varepsilon_{\mathbf{p}+\mathbf{k}}}\right)\right]
\end{aligned}
$$

and

$$
\begin{aligned}
F_{3}\left(q, \alpha_{m} ; k, \omega_{n}\right)= & 2 \int \frac{d^{3} p}{(2 \pi)^{3}} f\left(\varepsilon_{\mathbf{p}}\right) \\
& \times\left[\frac{1}{\omega_{n}+\varepsilon_{\mathbf{p}}-\varepsilon_{\mathbf{p}+\mathbf{k}}} \frac{1}{\omega_{n}+\alpha_{m}+\varepsilon_{\mathbf{p}}-\varepsilon_{\mathbf{p}+\mathbf{k}+\mathbf{q}}}\right. \\
& +\frac{1}{-\omega_{n}+\varepsilon_{\mathbf{p}}-\varepsilon_{\mathbf{p}+\mathbf{k}}} \frac{1}{\alpha_{m}+\varepsilon_{\mathbf{p}}-\varepsilon_{\mathbf{p}-\mathbf{q}}} \\
& +\frac{1}{-\left(\omega_{n}+\alpha_{m}\right)+\varepsilon_{\mathbf{p}}-\varepsilon_{\mathbf{p}+\mathbf{k}+\mathbf{q}}} \\
& \left.\times \frac{1}{-\alpha_{m}+\varepsilon_{\mathbf{p}}-\varepsilon_{\mathbf{p}+\mathbf{q}}}\right] .
\end{aligned}
$$

Here $f\left(\varepsilon_{\mathbf{p}}\right)$ is the Fermi function. It is important to note that in the limit $T \rightarrow 0$ the $\mathbf{p}$ integration can be performed by analytical means, i.e., $F_{1}, F_{2}$, and $F_{3}$ can be represented in terms of elementary functions. Before this is done the $m$ summation over the (internal) imaginary frequencies is replaced by a contour integration and the result is then continued into the upper complex $\omega$ plane. We have to perform the internal frequency integration and the $\mathbf{q}$ integration numerically. Using cylindrical coordinates with $\mathbf{k}$ as polar axis the angular integration is immediate and the numerical work is reduced to three-dimensional integrations. The details of the evaluation of the diagrams are outlined in the Appendix.

\section{RESULTS}

Here we discuss in detail our numerical results for $\operatorname{Im} \epsilon(k, \omega)$ obtained for arbitrary $(k, \omega)$ outside the ph spectrum. To demonstrate their importance we consider the bandstructure contributions to $\operatorname{Im} \epsilon(\mathbf{k}, \omega)$ for $\mathrm{Al}$ which we calculate within the RPA in second order in the weak pseudopotential. We compare the results with $\operatorname{Im} \epsilon_{A B C}(k, \omega)$ evaluated at the appropriate electron density of Al. Finally we calculate the imaginary part of the local-field factor $\operatorname{Im} G(k, \omega)$.

To gain confidence in our results we first derive an asymptotic high-frequency limit and make contact with results in the literature obtained in limiting cases. We use wave numbers in units of the Fermi wave number $k_{F}$ related to the electron density by $n_{0}=k_{F}^{3} / 3 \pi^{2}$, and frequencies in units of the Fermi frequency $\omega_{F}=\varepsilon_{F} / \hbar$ with $\varepsilon_{F}=\hbar^{2} k_{F}^{2} / 2 m . \omega_{p}$ $=\sqrt{4 \pi n_{0} e^{2} / m}$ is the plasma frequency, which in our units is defined by $\omega_{p}=2 \sqrt{3} \alpha^{2} \sqrt{r_{s}} \approx 0.9405 \sqrt{r_{s}}$ with $\alpha=(4 / 9 \pi)^{1 / 3}$ $\approx 0.5211$ and $4 \pi\left(r_{s} a_{0}\right)^{3} / 3=1 / n_{0}$ ( $a_{0}$ Bohr radius).

In the limit of high frequencies, i.e., $\omega \gg k^{2}+2 k$, screening is unimportant, and $\operatorname{Im} \pi_{A B}(k, \omega) \equiv \operatorname{Im}\left[\pi_{A}(k, \omega)\right.$ $\left.+\pi_{B}(k, \omega)\right]$ and $\operatorname{Im} \pi_{C}(k, \omega)$ can be evaluated analytically after expanding the expression for $\operatorname{Im} \pi_{A B}(k, \omega)$ to second order in the Coulomb potential. Thus we use the approximation

$$
\frac{v(q)}{\epsilon_{L}\left(q, \omega^{\prime}\right)} \approx v(q)-v(q)^{2} \pi_{0}\left(q, \omega^{\prime}\right)
$$

Only the second term of Eq. (3.1) contributes and we find 


$$
\begin{aligned}
& v(k) \operatorname{Im} \pi_{A B}(k, \omega)=\frac{\pi}{8 \sqrt{2}} \frac{\omega_{p}^{6}}{\omega^{9 / 2}}\left(1+\frac{27}{4} \frac{k^{2}}{\omega}+\cdots\right), \\
& v(k) \operatorname{Im} \pi_{C}(k, \omega)=-\frac{\pi}{8 \sqrt{2}} \frac{\omega_{p}^{6}}{\omega^{9 / 2}}\left(1+\frac{43}{20} \frac{k^{2}}{\omega}+\cdots\right) .
\end{aligned}
$$

Equations (3.2) and (3.3) demonstrate once more that $\pi_{C}(k, \omega)$ must be included to obtain a physically meaningful result because in the sum the $k$-independent terms must cancel and we obtain

$$
\operatorname{Im} \epsilon_{A B C}(k, \omega)=\frac{23 \pi}{40 \sqrt{2}} \frac{\omega_{p}^{6}}{\omega^{11 / 2}} k^{2} .
$$

While this result is an important test for our numerical results for arbitrary $k$ and sufficiently large $\omega$, another important asymptotic limit is the result of Hasegawa and Watabe quoted above which is valid in the limit $k \rightarrow 0$ and $\omega>0$. It can be written in the form

$$
\operatorname{Im} \epsilon_{A B C}(k, \omega)=\frac{\omega_{p}^{4}}{\omega^{4}} k^{2} \gamma_{H W}^{\prime \prime}(\omega),
$$

where

$$
\begin{aligned}
\gamma_{H W}^{\prime \prime}(\omega)= & \frac{1}{10 \pi \omega^{2} \omega_{p}^{2}} \int_{0}^{\infty} d q q^{2} \int_{0}^{\omega} d \omega^{\prime}\left[16\left(\omega-\omega^{\prime}\right)^{2}\right. \\
& \times \operatorname{Im}\left(\frac{-1}{\epsilon_{L}\left(q, \omega^{\prime}\right)}\right) \operatorname{Im} \epsilon_{T}\left(q, \omega-\omega^{\prime}\right) \\
& \left.+23 \omega^{2} \operatorname{Im}\left(\frac{-1}{\epsilon_{L}\left(q, \omega^{\prime}\right)}\right) \operatorname{Im}\left(\frac{-1}{\epsilon_{L}\left(q, \omega-\omega^{\prime}\right)}\right)\right]
\end{aligned}
$$

$\epsilon_{L}(q, \omega)$ and $\epsilon_{T}(q, \omega)$ are the well-known longitudinal and transverse Lindhard dielectric functions. ${ }^{18}$ The second term gives the leading contribution at $\omega \gg \omega_{p}$. Making use of

$$
\operatorname{Im}\left[-1 / \epsilon_{L}(q, \omega)\right] \rightarrow \operatorname{Im} \epsilon_{L}(q, \omega) \rightarrow \frac{\pi \omega_{p}^{2}}{2 q^{2}} \delta\left(\omega-q^{2}\right)
$$

the integrations can be performed easily, and we find again Eq. (3.4). The $\omega$ dependence in Eq. (3.4) was also found by Glick and Long, ${ }^{7}$ but their prefactor was half as large. We shall comment on this later.

We now turn to the case of arbitrary $(k, \omega)$ above and below the ph excitations. Comparison of the formula of HW with our derivation for arbitrary $(k, \omega)$ outlined in the Appendix shows that the calculation for finite $k$ is much more complicated, and a comparison of our numerical results with the results from the HW formula provides an important test of our calculation. For the discussion of these results it is convenient to define a function $\gamma^{\prime \prime}(k, \omega)$ by

$$
\operatorname{Im} \epsilon_{A B C}(k, \omega)=\frac{\omega_{p}^{4}}{\omega^{4}} k^{2} \gamma^{\prime \prime}(k, \omega) .
$$

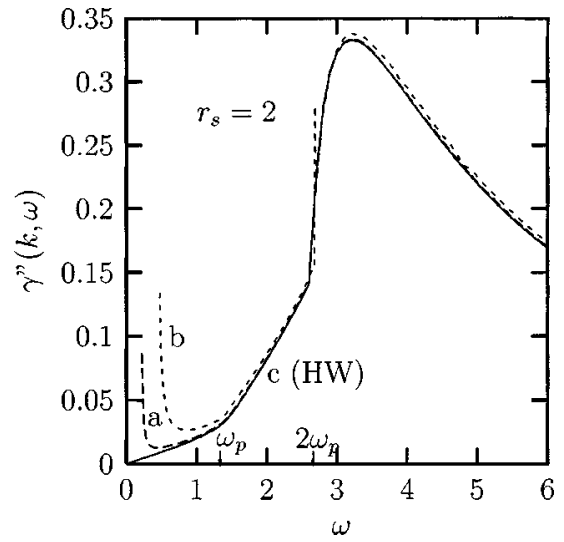

FIG. 2. Comparison of $\gamma^{\prime \prime}(k, \omega)$ at a, $k=0.1$, and $\mathrm{b}, k=0.2$, with c, $\gamma_{H W}^{\prime \prime}(\omega)=\gamma^{\prime \prime}(0, \omega)$.

In Fig. 2 we demonstrate that in the limit of small $k$, $\gamma^{\prime \prime}(k, \omega) \rightarrow \gamma_{H W}^{\prime \prime}(\omega)$. At finite $k$ the sudden rise towards small $\omega$ signals the onset of ph excitations below $\omega=k^{2}+2 k$. (The logarithmic singularity at $\omega=k^{2}+2 k$ is a well-known artifact of perturbation theory.)

In general, the excitation spectrum manifest in $\operatorname{Im} \epsilon_{A B C}(k, \omega)$ or $\gamma^{\prime \prime}(k, \omega)$ consists of two-particle-hole pair (ph-ph) excitations, two-plasmon (pl-pl) excitations, and combined particle-hole pair and plasmon (ph-pl) excitations. The last two are consequences of dynamical screening of the Coulomb interaction. We now discuss these individual contributions by showing how they evolve with increasing $k$ from the result of $\mathrm{HW}$.

\section{A. Plasmon-plasmon excitations}

$\mathrm{GV}$ pointed out that in the high-density limit the correct $r_{s}$ dependence of the compressibility sum rule is guaranteed only if the Coulomb interaction is screened dynamically and if the $\mathrm{C}$ diagrams are taken into account. Since the $\mathrm{C}$ diagrams are of second order in the dynamically screened Coulomb interaction they necessarily contain pl-pl excitations. While in the limit $k \rightarrow 0$ pl-pl excitations give a rather smooth contribution to $\gamma^{\prime \prime}(0, \omega)=\gamma_{H W}^{\prime \prime}(\omega)$, for finite $k$ they develop a peak that is visible as a needlelike structure in $\gamma^{\prime \prime}(k, \omega)$ close to $2 \omega_{p}$ in Fig. 2.

As shown in Fig. 3 the pl-pl peak increases in height and width, and shifts to higher frequencies with increasing $k$. The sharp onset and the upper limit of pl-pl excitations can be estimated from the plasmon poles of

$$
\begin{aligned}
& \operatorname{Im}\left[U_{\mathbf{q}}(x+i \boldsymbol{\epsilon})\right] \operatorname{Im}\left[U_{\mathbf{q}+\mathbf{k}}(y+i \delta)\right] \\
&= v(q) v(|\mathbf{q}+\mathbf{k}|) \operatorname{Im}\left[-1 / \epsilon_{L}(q, x)\right] \\
& \times \operatorname{Im}\left[-1 / \epsilon_{L}(|\mathbf{k}+\mathbf{q}|, y)\right]
\end{aligned}
$$

appearing in one of the expressions for $\operatorname{Im} \pi_{C}(k, \omega)$ (see Appendix), with $y=\omega-x$. Explicitly, the plasmon poles are given by

$$
\operatorname{Im}\left[-1 / \epsilon_{L}(q, x)\right]_{p l}=\pi \delta\left(x-\omega_{p}(q)\right)\left[\partial \epsilon_{L}(q, x) / \partial x\right]_{x=\omega_{p}(q)}^{-1}
$$

and 


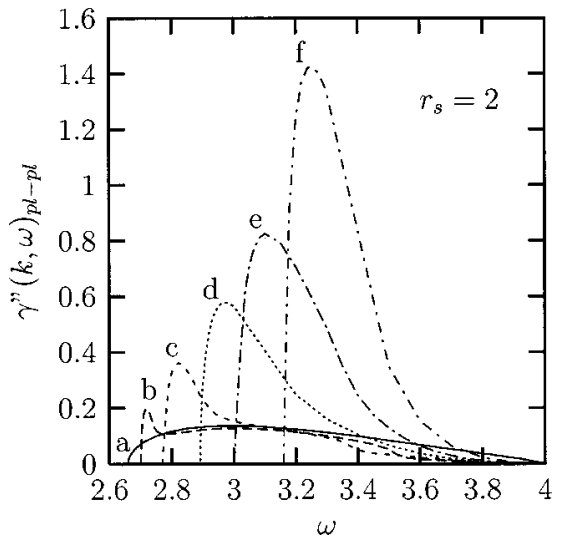

FIG. 3. Contribution from two-plasmon excitations $\gamma_{p l-p l}^{\prime \prime}(k, \omega)$ for various $k<2 q_{c}$, a: $k=0(\mathrm{HW}) ; \mathrm{b}: k=0.3$; c: $k=0.5 ; \mathrm{d}: k$ $=0.7 ; \mathrm{e}: k=0.85 ; \mathrm{f}: k=1.0$.

$$
\begin{aligned}
\operatorname{Im}[ & \left.-1 / \epsilon_{L}(|\mathbf{q}+\mathbf{k}|, y)\right]_{p l} \\
& =\pi \delta\left(y-\omega_{p}(|\mathbf{q}+\mathbf{k}|)\left[\partial \epsilon_{L}(|\mathbf{q}+\mathbf{k}|, y) / \partial y\right]_{y=\omega_{p}(|\mathbf{q}+\mathbf{k}|)}^{-1},\right.
\end{aligned}
$$

where $x=\omega_{p}(q)$ and $y=\omega_{p}(|\mathbf{q}+\mathbf{k}|)$ are the solutions of $\epsilon_{L}(q, x)=0$ and $\epsilon_{L}(|\mathbf{q}+\mathbf{k}|, y)=0$. On performing the integration over the internal frequency $x$, the product of the two $\delta$ functions gives

$$
\delta\left[\omega-\omega_{p}(q)-\omega_{p}(|\mathbf{q}+\mathbf{k}|)\right] .
$$

Since plasmons can exist only for $q \leqslant q_{c}$ and $|\mathbf{q}+\mathbf{k}| \leqslant q_{c}$, where $q_{c}$ is the RPA plasmon cutoff wave number with $\omega_{p}\left(q_{c}\right)=q_{c}^{2}+2 q_{c}$, it follows that pl-pl excitations are possible for

$$
0 \leqslant k \leqslant 2 q_{c},
$$

and we find that the onset of pl-pl excitations is determined by (see Fig. 4)

$$
\omega_{p l-p l}^{\text {onset }}(k)=2 \omega_{p}(k / 2) .
$$

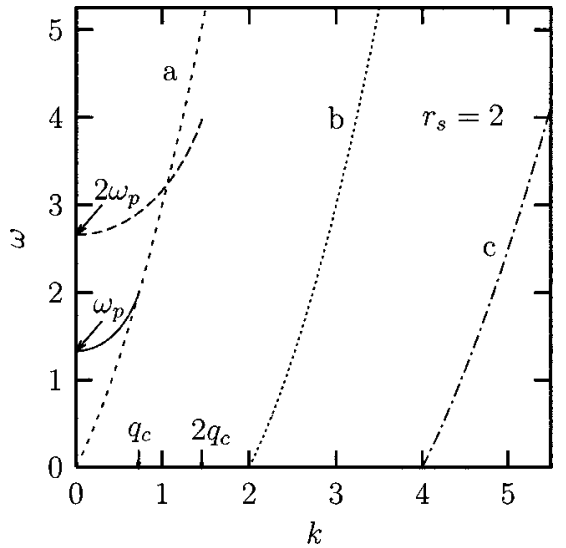

FIG. 4. Excitation spectrum of the homogeneous electron gas; a: upper bound of the ph spectrum $\omega=k^{2}+2 k$; b: lower bound of the ph spectrum $\omega=k^{2}-2 k$; c: lower bound of the ph-ph spectrum $\omega$ $=k^{2} / 2-2 k . \omega_{p}$ denotes the onset of the RPA plasmon dispersion $\omega_{p}(k)$ with the cutoff wave number $q_{c}$. The line $2 \omega_{p}(k / 2)$ marks the onset of pl-pl excitations.

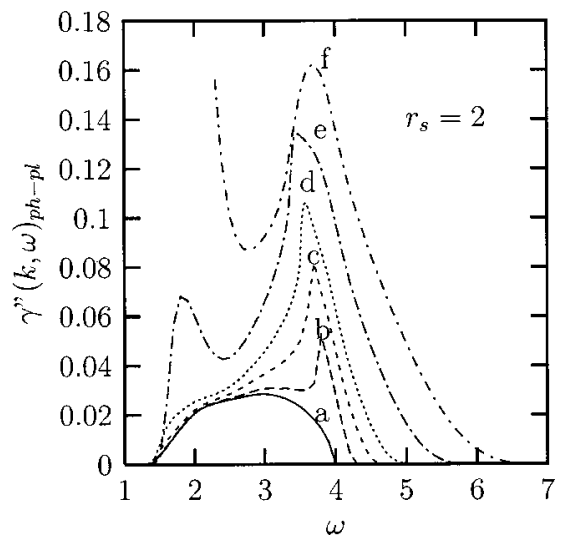

FIG. 5. Contribution from combined particle-hole pair plasmon excitations $\gamma_{p h-p l}^{\prime \prime}(k, \omega)$ for various $k, \mathrm{a}: k=0(\mathrm{HW})$; b: $k=0.1$, c: $k=0.2$; d: $k=0.3$; e: $k=0.5$; f: $k=0.7$.

For small $k \omega_{p}(k)=\omega_{p}+2 \alpha k^{2}$ with the small $k$ plasmon dispersion constant $\alpha=0.6 / \omega_{p}$ (in our units), and we have

$$
\omega_{p l-p l}^{\text {onset }}(k)=2 \omega_{p}+\alpha k^{2} .
$$

The upper limit for pl-pl excitations at any allowed $k$ is determined by $2 \omega_{p}\left(q_{c}\right)=2\left(q_{c}^{2}+2 q_{c}\right)$. For $r_{s}=2$ the plasmon cutoff wave number $q_{c} \approx 0.73$ and $2 \omega_{p}\left(q_{c}\right) \approx 4$.

The position of the maximum is a combined effect of the Coulomb potential factors and the $\delta$ functions from the plasmon poles. At small $k$ it can be estimated from the maximum of the integral

$$
\begin{aligned}
I & =\int d^{3} q \frac{1}{q^{2}} \frac{1}{|\mathbf{q}+\mathbf{k}|^{2}} \delta\left(\omega-2 \omega_{p}-2 \alpha\left[q^{2}+|\mathbf{q}+\mathbf{k}|^{2}\right]\right) \\
& =\frac{\pi}{4 \alpha k a} \ln \left|\frac{a+k \sqrt{a-k^{2} / 4}}{a-k \sqrt{a-k^{2} / 4}}\right|,
\end{aligned}
$$

where $a=\left[\omega-2 \omega_{p}\right] / 4 \alpha$ and we find

$$
\omega_{p l-p l}^{\max }(k) \approx 2 \omega_{p}+2 \alpha k^{2} .
$$

Although in principle pl-pl excitations are possible for any $k \leqslant 2 q_{c}$, above a certain $k_{1}$ they fall partly into the ph spectrum as is demonstrated in Fig. 4. $k_{1}$ is determined from $k^{2}$ $+2 k=2 \omega_{p}(k / 2) \approx 2 \omega_{p}+\alpha k^{2}$. For $r_{s}=2$ one obtains $k_{1}$ $\approx 1.03$ from the approximate equation, very close to the exact value. Above $k_{2}$ determined from $k^{2}+2 k=2 \omega_{p}\left(q_{c}\right)$ $=2\left(q_{c}^{2}+2 q_{c}\right)$ all pl-pl excitations lie inside the ph spectrum. (For $r_{s}=2, k_{2} \approx 1.23$.)

\section{B. Particle-hole pair-plasmon excitations}

While pl-pl excitations are restricted to $k<2 q_{c}$, ph-pl excitations are possible at any $k$. They arise again from $d y$ namical screening of the Coulomb interaction but now in both $\operatorname{Im} \pi_{A B}(k, \omega)$ and $\operatorname{Im} \pi_{C}(k, \omega)$. Their contributions at various $k$ as a function of frequency are illustrated in Fig. 5.

These excitations begin at $\omega=\omega_{p}$ for $k \leqslant k_{0}$, where $k_{0}$ is determined from $\omega_{p}=k^{2}+2 k$, while for $k \geqslant k_{0}$ they partly lie inside the ph spectrum. Their maximum frequency is determined by the highest frequency of the plasmon and the high- 


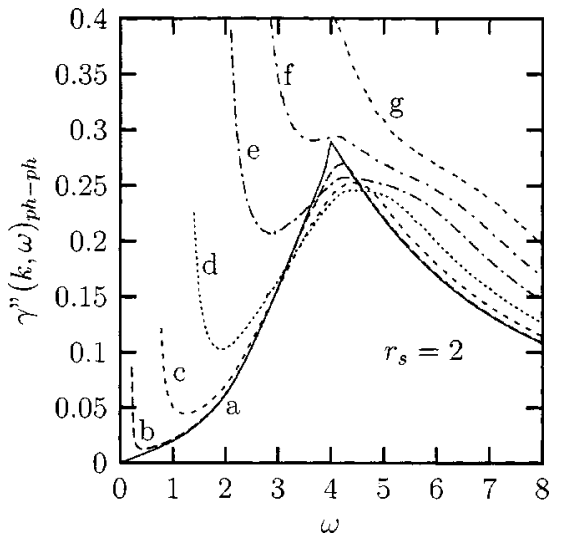

FIG. 6. Contribution from two-particle-hole pair excitations $\gamma_{p h-p h}^{\prime \prime}(k, \omega)$ for various $k$, a: $k=0(\mathrm{HW}) ; \mathrm{b}: k=0.1 ; \mathrm{c}: k=0.3$; d: $k=0.5$; e: $k=0.7$; f: $k=0.85$; g: $k=1.0$.

est frequency of ph excitation related to $q=q_{c}$ and to $|\mathbf{q}+\mathbf{k}|=q_{c}+k$. Since both are located on the upper boundary of the ph spectrum we find

$$
\omega_{p h-p l}^{u p p e r}(k)=\left(k+q_{c}\right)^{2}+2\left(k+q_{c}\right)+q_{c}^{2}+2 q_{c} .
$$

\section{Particle-hole pair-particle-hole pair excitations}

These excitations are possible for any $(k, \omega)$ above the ph spectrum. The variation of $\gamma_{p h-p h}^{\prime \prime}(k, \omega)$ as a function of $\omega$ with increasing $k$ is shown in Fig. 6.

For $\omega<\omega_{p}$ ph-ph excitations are the only excitations, i.e., $\gamma^{\prime \prime}(k, \omega)=\gamma_{p h-p h}^{\prime \prime}(k, \omega)$. There is no upper frequency limit as is clear from the asymptotic limit Eq. (3.4), but ph-ph excitations occur below the ph spectrum for $k \geqslant 2$, provided

$$
\omega>\left(k^{2} / 2-2 k\right) \Theta(k-4),
$$

as predicted on general grounds in Ref. 19. Results in terms of $\operatorname{Im} \epsilon_{A B C}(k, \omega)$ as a function of $\omega$ for values of $(k, \omega)$ in the relevant regime are shown in Fig. 7. A monotonic increase is observed from $\omega=0$ to the lower bound of the ph spectrum given by $\omega=\left(k^{2}-2 k\right) \Theta(k-2)$ (where again an unphysical logarithmic singularity occurs).

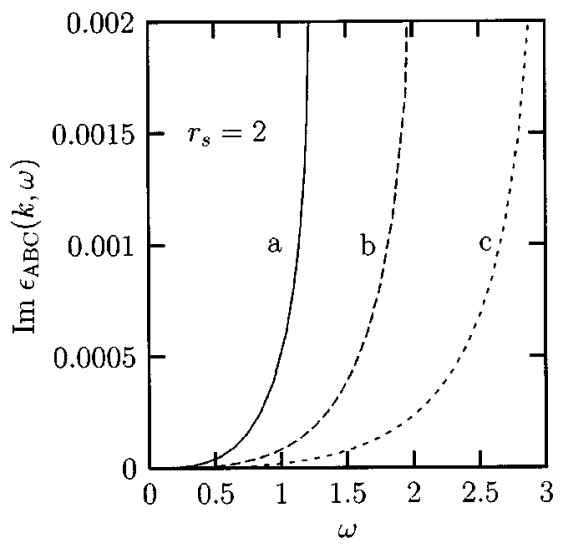

FIG. 7. $\operatorname{Im} \epsilon_{A B C}(k, \omega)$ below the ph spectrum $(k>2)$, a: $k$ $=2.5$; b: $k=2.75$, c: $k=3.00$.

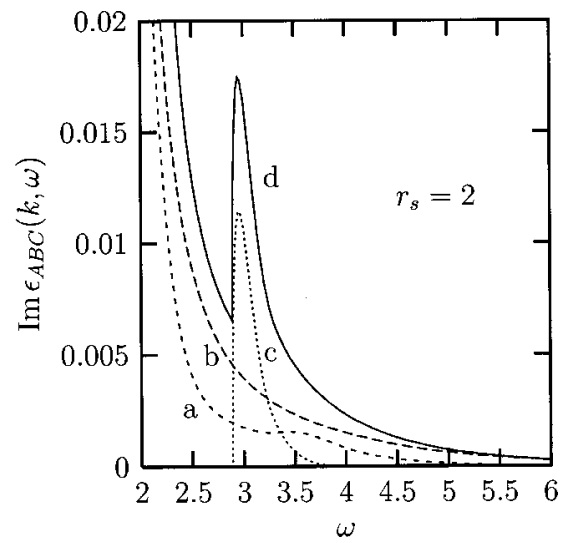

FIG. 8. $\operatorname{Im} \epsilon_{A B C}(k, \omega)$ for $k=0.7$ as a function of $\omega$, a: ph-pl contribution; b: ph-ph contribution; c: pl-pl contribution; d: total sum.

\section{Im $\epsilon_{A B C}(k, \omega)$ and comparison with crystal potential effects}

The relative importance of the three contributions depends strongly on $(k, \omega)$. This is illustrated in Fig. 8, where for $k=0.7$ the individual contributions to $\operatorname{Im} \epsilon_{A B C}(k, \omega)$ and their sum are shown.

Above the ph spectrum, ph-ph excitations give rise to a structureless background. They are possible for any $k$. ph-pl excitations are also possible for any $k$, but limited to a finite frequency interval, and the magnitude of their contribution is mostly smaller than that of the ph-ph excitation but of the same order. The phase space of pl-pl excitations is rather limited, but these excitations cause a prominent structure of $\operatorname{Im} \epsilon_{A B C}(k, \omega)$, whereas little structure from the ph-pl contribution survives in the total sum (see Fig. 9).

We now discuss the relative importance of dynamical correlation effects consisting of multiple excitations. In simple metals such as Al which are nature's closest realization of the model of a homogeneous electron gas, there is an additional contribution to $\operatorname{Im} \epsilon(\mathbf{k}, \omega)$. The crystal potential gives rise to interband transitions and local-field effects due to the inhomogeneity (outside the ph excitation spectrum of the homogeneous electron gas). In nearly free electron (NFE) pseudopotential theory the band structure (bs) contribution to $\operatorname{Im} \epsilon(\mathbf{k}, \omega)$ can be calculated within the RPA by perturbation theory ${ }^{20}$ to second order in the screened pseudopotential:

$$
\begin{aligned}
& \operatorname{Im} \epsilon_{b s}(\mathbf{k}, \omega) \\
& \quad=\operatorname{Im}\left[\epsilon(\mathbf{k}, \mathbf{k}, \omega)-\sum_{\mathbf{G} \neq 0} \frac{\epsilon(\mathbf{k}, \mathbf{k}+\mathbf{G}, \omega) \epsilon(\mathbf{k}+\mathbf{G}, \mathbf{k}, \omega)}{\epsilon_{L}(|\mathbf{k}+\mathbf{G}|, \omega)}\right] .
\end{aligned}
$$

Here $\epsilon\left(\mathbf{k}+\mathbf{G}, \mathbf{k}+\mathbf{G}^{\prime}, \omega\right)$ are elements of the dielectric matrix. $^{21}$ The diagonal element is evaluated in second order and the off-diagonal elements to first order in the pseudopotential.

$\mathrm{Al}$ is an ideal candidate because it has a rather high density $\left(r_{s}=2.075\right)$ and its pseudopotential is known to be rather weak. In the following we compare effects of the pseudopotential and dynamical correlations on $\operatorname{Im} \epsilon(\mathbf{k}, \omega)$, using an Ashcroft empty core potential. Band-structure effects are dominant at small $k(k \ll 1)$, but with increasing $k$ 

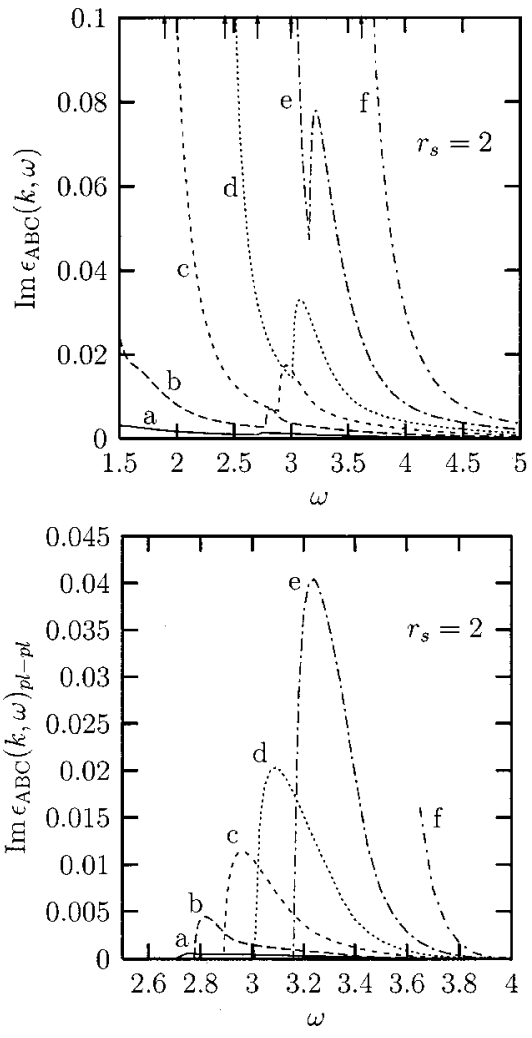

FIG. 9. Upper part: $\operatorname{Im} \epsilon_{A B C}(k, \omega)$ for various $k \neq 0$ as a function of $\omega$ above $\omega=k^{2}+2 k$ (denoted by arrows), a: $k=0.3$; b: $k$ $=0.5$; $\mathrm{c}: k=0.7$; $: k=0.85$; e: $k=1.0 ; \mathrm{f}: k=1.15$. Lower part: pl-pl contribution to $\operatorname{Im} \epsilon_{A B C}(k, \omega)$ for the same $k$ values. For $k=1.15$ part of the pl-pl contribution lies inside the ph spectrum.

correlation effects increase and for $k \geqslant 1$ prevail (see Fig. 10). The directional dependence of band structure effects is mostly rather small.

A simple addition of the two contributions is, however, inconclusive, because mixed terms that are first order in the screened pseudopotential and in the dynamically screened Coulomb potential must be included for consistency and completeness. These terms have not been considered so far and are beyond the scope of the present investigation.

\section{E. Results for different densities}

The diagrams in Fig. 1 represent the leading corrections to the RPA in a high-density expansion. From the investigation of the compressibility sum rule GV concluded that this low order perturbation expansion of $\pi_{p}(k, \omega)$ is not adequate for $r_{s} \geqslant 3$.

In the spirit of perturbation theory higher-order multiple excitations are probably the source of additional sizeable dynamical effects at lower density. Nevertheless, it is interesting to investigate trends within the present approximation. For this purpose we have calculated $\operatorname{Im} \epsilon_{A B C}$ for $r_{s}=4$ and $r_{s}=6$ as well as $r_{s}=2$. We have selected a $k$ regime that includes the effects arising from plasmons. Since the plasmon cutoff wave number $q_{c}$ depends on $r_{s}$ we determined the $k$ value in such a way that $k / q_{c}=$ const, here const $\approx 1.17$. Figure 11 shows a substantial increase of dynamical correlation effects with decreasing density even within the present approximation. Note that $\omega$ is in units of the $r_{s}$-dependent free-electron Fermi frequency $\omega_{F}$.
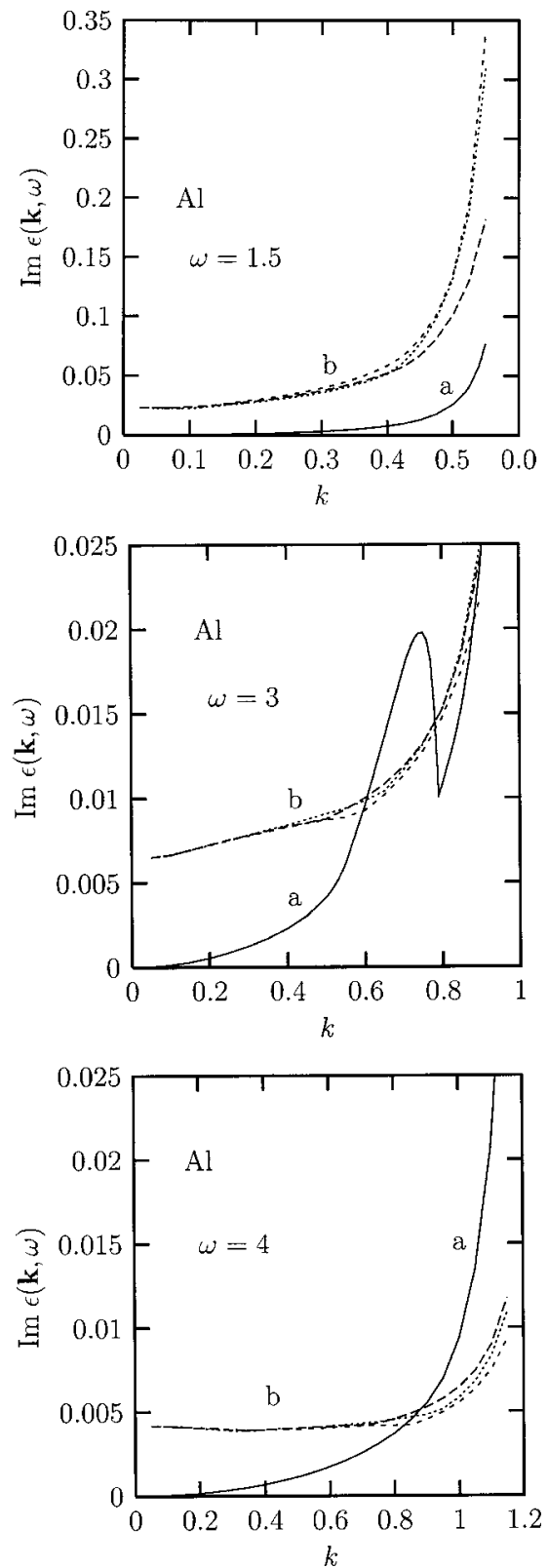

FIG. 10. Comparison of correlation and band-structure effects: $\operatorname{Im} \epsilon(\mathbf{k}, \omega)$ as a function of $\mathbf{k}$ at $\omega=1.5, \omega=3$, and $\omega=4$; a: $\operatorname{Im} \epsilon_{A B C}(k, \omega) ; \mathrm{b}: \operatorname{Im} \epsilon_{b s}(\mathbf{k}, \omega)$ along principal symmetry directions.

\section{F. Local field factor $G(k, \omega)$}

As pointed out in the introduction dynamical correlations are at the center of time-dependent density-functional theory, in which the general form of the exchange-correlation functional is unknown. Approximations are usually constructed from results for the homogeneous electron gas, but even for this model exact results are difficult to obtain in the case of TDFT.

It is common practice to account for dynamical exchange correlation effects in the dielectric function by introducing a local-field factor $G(k, \omega)$ [see Eq. (1.1) defined in the spirit of a generalized mean-field theory]. ${ }^{4}$ Very often phenomenological forms are adopted which are constructed by fitting parameters using sum rules, the fluctuation-dissipation theorem and asymptotic limits. In many cases the frequency dependence of $G(k, \omega)$ is neglected altogether. 


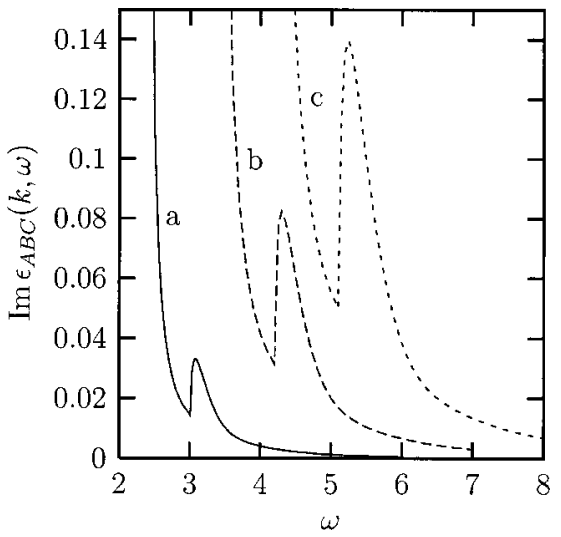

FIG. 11. Comparison of $\operatorname{Im} \epsilon_{A B C}(k, \omega)$ as a function of $\omega$ for different densities; a: $r_{s}=2, \quad k=0.85 ; \mathrm{b}: r_{s}=4, \quad k=1.1 ; \mathrm{c}: r_{s}$ $=6, k=1.28, \quad k$ is chosen to satisfy $k / q_{c} \approx$ const.

A formally exact definition in terms of the proper polarizability $\pi_{p}(k, \omega)$ follows from Eqs. (1.3) and (1.1):

$$
G(k, \omega)=\frac{1}{v(k)}\left(\frac{1}{\pi_{0}(k, \omega)}-\frac{1}{\pi_{p}(k, \omega)}\right) .
$$

Obviously the introduction of $G(k, \omega)$ does not lead to any new physics, it is a different way to represent corrections to the RPA. Here we present $\operatorname{Im} G(k, \omega)$ that can be useful for comparison with phenomenological forms that are obtained by first constructing the imaginary part such as the Padé-like interpolation formula of GKI. Since outside the ph spectrum $\operatorname{Im} \pi_{0}(k, \omega)$ vanishes, it follows that

$$
\operatorname{Im} G(k, \omega)=-\frac{1}{v(k)} \operatorname{Im}\left(\frac{1}{\pi_{p}(k, \omega)}\right) .
$$

From our results we obtain in the spirit of perturbation theory

$$
\operatorname{Im} G(k, \omega) \approx \frac{\operatorname{Im} \epsilon_{A B C}(k, \omega)}{\left[\epsilon_{L}(k, \omega)-1\right]^{2}}=\frac{\omega_{p}^{4}}{\omega^{4}} k^{2} \frac{\gamma^{\prime \prime}(k, \omega)}{\left[\epsilon_{L}(k, \omega)-1\right]^{2}} .
$$

Figure 12 demonstrates that $\operatorname{Im} G(k, \omega)$ increases with increasing $k$, as expected on general grounds.

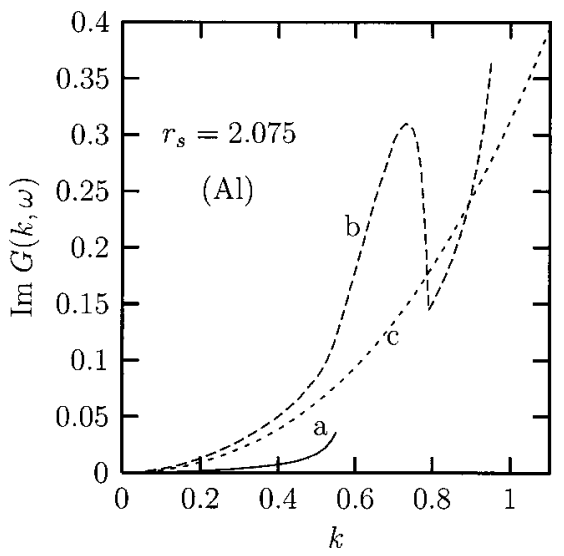

FIG. 12. $\operatorname{Im} G(k, \omega)$ as a function of $k$ at fixed frequencies: a: $\omega=1.5 ;$ b: $\omega=3$; c: $\omega=5$.

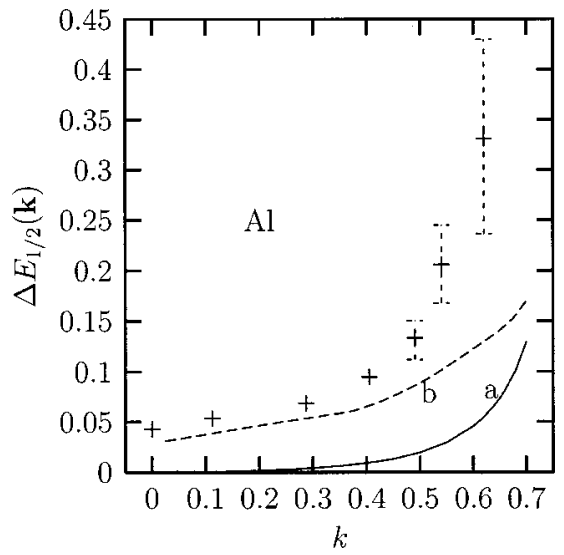

FIG. 13. Plasmon linewidth (FWHM) $\Delta E_{1 / 2}(\mathbf{k})$ of $\mathrm{Al}$ in units of $\varepsilon_{F}$, a: due to multiple excitations; b: due to interband transitions and local-field effects for $\mathbf{k} \|(421)$ (to mimic polycrystalline $\mathrm{Al}$ ), symbols are experimental data on polycrystalline Al.

In the context of TDFT, GKI introduced the so-called exchange-correlation kernel $f_{x c}(k, \omega)$ related to $G(k, \omega)$ by

$$
f_{x c}(k, \omega)=-v(k) G(k, \omega) .
$$

For lack of a microscopic theory GKI constructed a Padè approximation for $f_{x c}(k \rightarrow 0, \omega)$ with parameters fitted by using the compressibility and third frequency moment sum rule and the high-frequency limit for $\operatorname{Im} \epsilon(k, \omega)$ of Ref. 7.

\section{APPLICATIONS}

Now we discuss some consequences of $\operatorname{Im} \epsilon_{A B C}(k, \omega)$ on observable properties of simple metals such as the plasmon damping, the high-frequency tail of the dynamic structure factor and the optical absorption.

\section{A. Plasmon damping}

While in the RPA the plasmon of the homogeneous electron gas is undamped, dynamical correlations such as multiple excitations considered here provide a decay mechanism of the plasmon for $0<k \leqslant q_{c}$. In real systems such as simple metals the plasmon can also decay via interband transitions and local-field effects as a consequence of the periodic crystal potential.

Treating correlation effects or band-structure effects as a small correction to the RPA of the homogeneous electron gas, the full width at half maximum (FWHM) $\Delta E_{1 / 2}(\mathbf{k})$ of the energy-loss function $\operatorname{Im}[-1 / \epsilon(\mathbf{k}, \omega)]$ is determined by

$$
\Delta E_{1 / 2}(\mathbf{k})=2\left(\operatorname{Im} \epsilon(\mathbf{k}, \omega)\left|\frac{\partial \epsilon_{L}(k, \omega)}{\partial \omega}\right|^{-1}\right)_{\omega=\omega_{p}(k)},
$$

where $\operatorname{Im} \epsilon(\mathbf{k}, \omega)$ is either $\operatorname{Im} \epsilon_{b s}(\mathbf{k}, \omega)$ or $\operatorname{Im} \epsilon_{A B C}(k, \omega)$.

In Fig. 13, $\Delta E_{1 / 2}(\mathbf{k})$ due to the decay by ph-ph and ph-pl excitations is compared with experimental data for $\mathrm{Al}$ and with $\Delta E_{1 / 2}(k)$ calculated within the nearly free-electron pseudopotential approximation in the RPA. (Since pl-pl excitations occur only for $\omega>2 \omega_{p}(k / 2)$ their frequency is too high to contribute to the decay.) Obviously the decay via interband transitions dominates. Only close to $q_{c}$ is the decay 

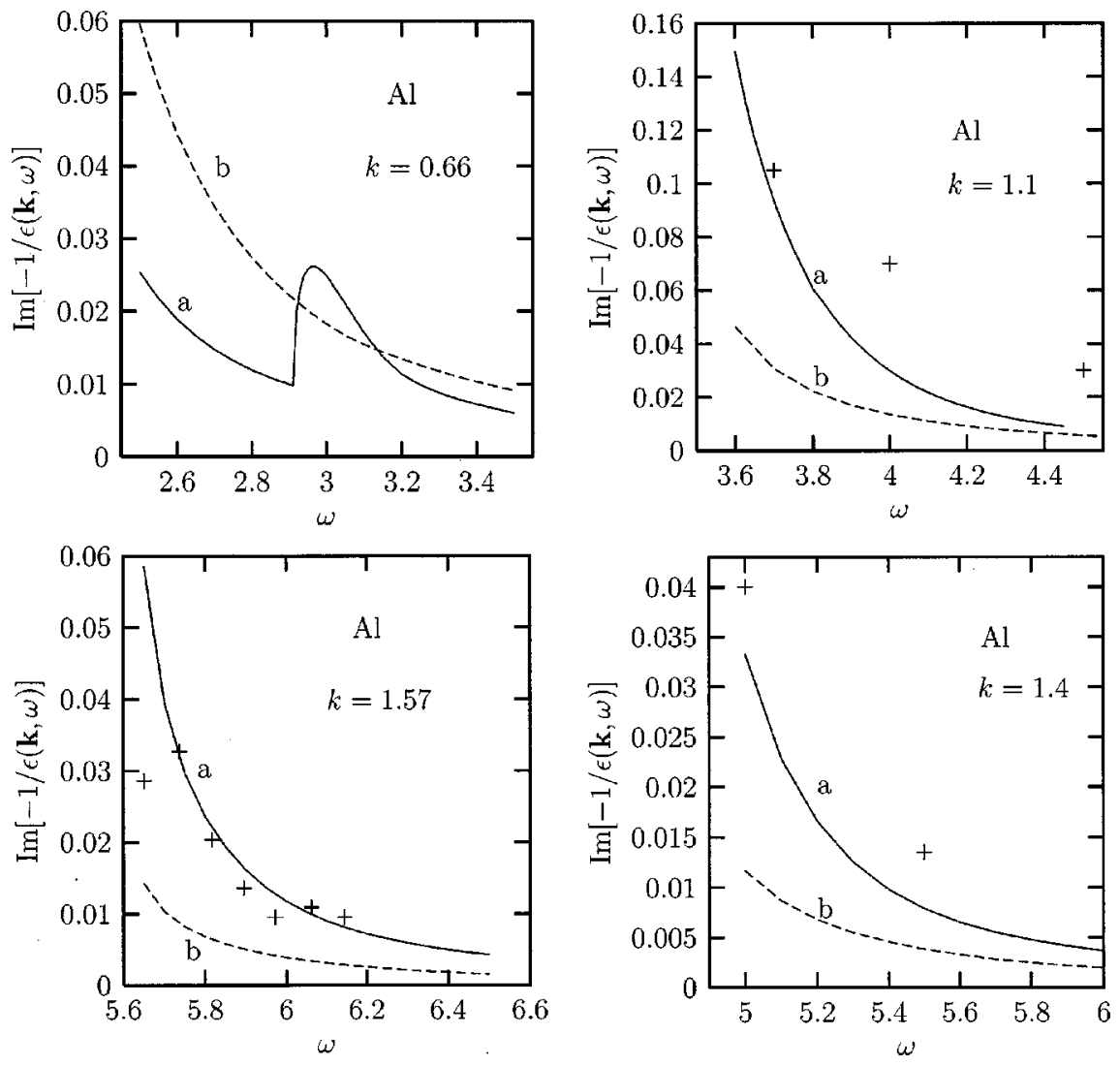

FIG. 14. High-frequency tail of $\operatorname{Im}[-1 / \epsilon(\mathbf{k}, \omega)]$ for $r_{s}$ $=2.075(\mathrm{Al})$ at $k=0.66, \quad k$ $=1.57(\mathbf{k} \|[100]) \quad$ and $\quad k$ $=1.1, \quad k=1.4(\mathbf{k} \|[130])$, a: due to dynamical correlations; b: due to band-structure effects. Symbols: experimental data (see text).

by ph-ph and ph-pl excitations sizeable. If we tentatively add the correlation contribution to the band-structure contribution it improves the agreement with experiment. ${ }^{22}$

\section{B. High-frequency tail of $S(\mathrm{k}, \omega)$}

The band-structure and dynamical correlation effects that cause plasmon damping are also responsible for a highfrequency tail of the dynamical structure factor $S(\mathbf{k}, \omega)$ which is observed experimentally at large $k$ in materials such as Al. By high frequency we mean frequencies above the upper bound of ph spectrum $\omega=k^{2}+2 k . S(\mathbf{k}, \omega)$ is related to the energy-loss function $\operatorname{Im}[-1 / \epsilon(\mathbf{k}, \omega)]$ by the fluctuation-dissipation theorem, i.e.,

$$
S(\mathbf{k}, \omega)=\frac{\hbar k^{2}}{4 \pi^{2} e^{2} n_{0}} \operatorname{Im}\left(\frac{-1}{\epsilon(\mathbf{k}, \omega)}\right) .
$$

Treating again correlation effects and band-structure effects as small corrections to the RPA of the homogeneous electron gas, we compare the two contributions to the loss function

$$
\frac{\operatorname{Im} \epsilon_{A B C}(k, \omega)}{\left|\epsilon_{L}(k, \omega)\right|^{2}}
$$

and

$$
\frac{\operatorname{Im} \epsilon_{b s}(\mathbf{k}, \omega)}{\left|\epsilon_{L}(k, \omega)\right|^{2}} .
$$

Since $1 / \epsilon_{L}(k, \omega)$ is real and rather structureless above the plasmon pole, the pl-pl peak in $\operatorname{Im} \epsilon_{A B C}(k, \omega)$ also appears in the loss function. Apart from a negligibly small anisotropy,
$\operatorname{Im} \epsilon_{b s}(\mathbf{k}, \omega)$ does not show any structure and the pl-pl peak should be experimentally observable in a simple metal such as Al. Indeed, in Fig. 2 of Ref. 23 in the $\omega$ plot of $S(\mathbf{k}, \omega)$ at $k=0.66$ (=0.61 a.u.) there is a structure of the right order of magnitude when compared with the theoretical prediction shown in Fig. 14. While theoretically the maximum occurs at about $35 \mathrm{eV}$, experimentally it is observed at about $31 \mathrm{eV}$. A downward shift of about $3 \mathrm{eV}$ can be estimated from the experimentally lower $\omega_{p} \approx 15 \mathrm{eV}$ and lower plasmon dispersion $\alpha \approx 0.3$. An additional example is provided by Ref. 24 . There the authors point out a shoulder on the high frequency side of $S(\mathbf{k}, \omega)$ for $k=1.1$ at $\omega \approx 3.44$ [see Fig. 1(b) of their work] that might be associated with pl-pl excitations. At this large $k$ the pl-pl peak merges with the main peak of $S(\mathbf{k}, \omega)$. Further experimental work with particular attention to the high-frequency tail of $S(\mathbf{k}, \omega)$ should help to clarify the situation.

At $k=0.66$ the correlation contribution is of the same order as the band structure contribution. When $k \geqslant 1$, however, the contribution from multiple excitations prevails (see Fig. 14). At large $k$ therefore the high-frequency tail of $S(\mathbf{k}, \omega)$ or $\operatorname{Im}[-1 / \epsilon(\mathbf{k}, \omega)]$, respectively, is caused essentially by multiple excitations. Comparison with experiments shows that bs effects alone are definitely too small to account for the observed magnitude of the tail. Correlation effects are much closer in magnitude as is shown by the experimental data for $k=1.57$ taken from Fig. 1 of Ref. 23 and for $k$ $=1.1$ and $k=1.4$ taken from Fig. 1 of Ref. 24 .

Note that errors of the magnitude of $S(\mathbf{k}, \omega)$ caused by normalization and smoothing of experimental curves affect the magnitude of the tails in particular. For example, we notice an unexpectedly large difference in the magnitude of 
the tails of $S(\mathbf{k}, \omega)$ at $k=1.34$ (1.24 a.u.) for $\mathbf{k} \|[100]$ and $\mathbf{k} \|[110]$ in Figs. 2 and 3 of Ref. 23, whereas the authors of Ref. 24 did not observe any directional dependence for $k$ $=1.4$.

\section{Optical absorption}

A homogeneous electron gas cannot absorb light, but the periodic potential in crystals makes the electronic system inhomogeneous and optical absorption is possible. The optical absorption is usually presented in terms of the optical conductivity $\sigma(\omega)$ and is related to the imaginary part of the transverse dielectric function $\operatorname{Im} \epsilon_{\perp}(\omega)$ via

$$
\sigma(\omega)=(\omega / 4 \pi) \operatorname{Im} \epsilon_{\perp}(\omega) .
$$

Ignoring for the moment intraband absorption due to phonon assisted transitions and following Hopfield, ${ }^{25} \operatorname{Im} \epsilon_{\perp}(\omega)$ due to the crystal potential can be written as

$$
\operatorname{Im} \epsilon_{\perp}(\omega)=\frac{4 \pi e^{2}}{m^{2} \omega^{4}} \sum_{\mathbf{G}, \mathbf{G}^{\prime}}(\vec{\eta} \mathbf{G})\left(\mathbf{G}^{\prime} \vec{\eta}\right) V_{-\mathbf{G}} V_{\mathbf{G}^{\prime}} \pi^{\prime \prime}\left(\mathbf{G}, \mathbf{G}^{\prime}, \omega\right)
$$

The Fourier coefficients of the (unscreened) crystal potential are denoted by $V_{\mathbf{G}}$, where $\mathbf{G}$ and $\mathbf{G}^{\prime}$ are reciprocal lattice vectors, $\vec{\eta}$ is the polarization vector of the electromagnetic field strength, and $\pi^{\prime \prime}\left(\mathbf{G}, \mathbf{G}^{\prime}, \omega\right)$ is the dissipative part of the electronic density-density response function. For the monovalent bcc alkali metals we use the NFE pseudopotential approximation and obtain to second order

$$
\begin{aligned}
\operatorname{Im} \epsilon_{\perp}(\omega) & =\frac{4}{3} \sum_{\mathbf{G}} \frac{\left|V_{\mathbf{G}}\right|^{2} G^{4}}{\omega^{4}} \operatorname{Im}\left[\frac{-1}{\epsilon(G, \omega)}\right] \\
& \approx \frac{4}{3} \sum_{\mathbf{G}} \frac{\left|V_{\mathbf{G}}^{S}\right|^{2} G^{4}}{\omega^{4}} \operatorname{Im} \epsilon(G, \omega) .
\end{aligned}
$$

$\epsilon(G, \omega)$ is now the dielectric function of the homogeneous electron gas. For simplicity we have assumed a local pseudopotential, where $\left|V_{\mathbf{G}}^{S}\right|^{2}=\left|V_{\mathbf{G}}\right|^{2} /|\epsilon(G, \omega)|^{2}$ $\left.\approx\left|V_{\mathbf{G}}\right|^{2}|/| \epsilon(G, 0)\right|^{2}$ includes screening by $\epsilon(G, 0)$. In the RPA $\operatorname{Im} \epsilon(G, \omega)=\operatorname{Im} \epsilon_{L}(G, \omega)$, which is nonzero for $\omega$ $\geqslant G^{2}-2 G$, when $G \geqslant 2$. Since the modulus of the smallest reciprocal-lattice vector of the bcc alkali metals is $G_{110}$ $=2.28$ these (interband) transitions begin at $\omega \approx 0.64$. They augment the phonon assisted transitions usually accounted for in a Drude relaxation time approximation by

$$
\operatorname{Im} \epsilon_{\text {Drude }}(\omega)=\frac{\omega_{p}^{2}}{m_{o p t} \tau \omega^{3}},
$$

valid for $\omega \tau \gg 1$, where the relaxation time $\tau$ can be estimated from the dc conductivity. The optical mass $m_{o p t}$ in units of the free-electron mass is slightly larger than one (see Smith $^{26}$ ).

This picture is qualitatively correct, but the observed optical absorption prior to the onset of interband transitions is considerably larger than predicted by the simple Drude formula $\left(\right.$ see Smith $^{26}$ ). For $\omega \leqslant 0.64$, ph-ph excitations offer an additional absorption mechanism mediated by the crystal (pseudo)potential. Their effect can be estimated by
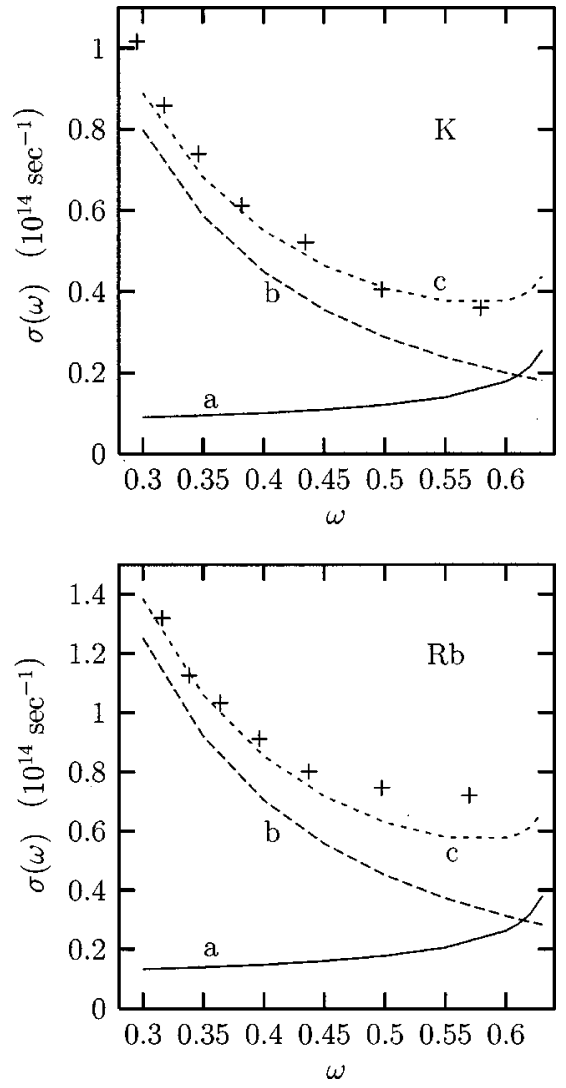

FIG. 15. $\sigma(\omega)$ for $\mathrm{K}$ and $\mathrm{Rb}$ below the onset of interband absorption at $\omega \approx 0.64$, a: due to ph-ph excitations mediated by the periodic potential; $b$ : Drude absorption; $\mathrm{c}$ : sum of $\mathrm{a}$ and $\mathrm{b}$, symbols are the experimental data of Smith (see text).

$$
\begin{aligned}
\operatorname{Im} \epsilon_{\perp}(\omega) & =\frac{4}{3} \sum_{\mathbf{G}} \frac{\left|V_{\mathbf{G}}^{S}\right|^{2} G^{4}}{\omega^{4}} \operatorname{Im} \epsilon_{A B C}(G, \omega) \\
& \approx 16 \frac{\left|V_{\mathbf{G}_{110}}^{S}\right|^{2} G_{110}^{4}}{\omega^{4}} \operatorname{Im} \epsilon_{A B C}\left(G_{110}, \omega\right) .
\end{aligned}
$$

Contributions associated with larger reciprocal-lattice vectors are negligibly small or zero because of Eq. (3.18). Although $\operatorname{Im} \epsilon_{A B C}\left(G_{110}, \omega\right)$ is rather small, the prefactor $\sim \omega^{-4}$ enhances the effect for $\omega<0.64$. The formula is valid for $\omega \tau \gg 1$.

We observe that close to the onset of interband transitions the contribution from ph-ph excitations is of the same order of magnitude as from the Drude term, if we use the same values for the pseudopotential coefficient that were used by Smith $^{26}$ to fit the interband absorption. This is demonstrated in Fig. 15 for $\mathrm{K}$ and $\mathrm{Rb}$. Of course its exact value depends crucially on the magnitude of $V_{\mathbf{G}_{110}}$. Moreover, the use of a local pseudopotential is questionable ${ }^{27}$ and a microscopic derivation of phonon assisted transitions close to the onset of interband transitions may deviate from the simple Drude formula. ${ }^{28}$ Nevertheless, our estimate shows that a complete theory of the optical absorption of the alkali metals must include dynamical correlations.

\section{SUMMARY AND CONCLUSIONS}

Within the model of the homogeneous electron gas we have calculated the leading corrections to the RPA bubble as 
defined by the diagrams in Fig. 1 and obtained $\operatorname{Im} \epsilon(k, \omega)$ for arbitrary $(k, \omega)$ outside the ph spectrum. In this way we have generalized the asymptotic $(k \rightarrow 0)$ result of HW, which was used by BCT to evaluate $f_{x c}(k=0, \omega)$.

Dynamical correlations that constitute $\operatorname{Im} \epsilon(k, \omega)$ outside the ph spectrum consist of multiple excitations such as phph, ph-pl, and pl-pl excitations. ph-ph excitations are possible for any $(k, \omega)$ above the ph spectrum. For $k>2$ they are the only mechanism for $\operatorname{Im} \epsilon(k, \omega)$ below the ph spectrum. There is, however, a lower bound defined for ph-ph excitations defined by $\omega=\left(k^{2} / 2-2 k\right) \Theta(k-4)$. pl-pl excitations are confined to $0<k \leqslant 2 q_{c}$ and $2 \omega_{p} \leqslant \omega \leqslant 2 \omega_{p}\left(q_{c}\right)$, and ph-pl excitations are possible for any $k$ and $\omega_{p} \leqslant \omega \leqslant(k$ $\left.+q_{c}\right)^{2}+2\left(k+q_{c}\right)+q_{c}^{2}+2 q_{c}$. There are no ph-pl and pl-pl excitations below the ph spectrum. For $\omega \gg k^{2}$ $+2 k \operatorname{Im} \epsilon(k, \omega)$ falls off as $\omega^{-11 / 2}$. The pl-pl excitations cause the dominant structure for $\omega>2 \omega_{p}(k / 2)$ and $0<k$ $<k_{1}$. Above this regime, $\operatorname{Im} \epsilon(k, \omega)$ falls off monotonically at a given $k$ with increasing frequency. At the boundaries of the ph excitations there is a logarithmic singularity that is a well known artifact of perturbation theory.

As expected on general grounds, dynamical correlations and their importance increase with increasing $k$ and for decreasing density, i.e., increasing $r_{s}$. The present calculation provides quantitative results based on a fully microscopic theory valid at high densities $\left(r_{s}<3\right)$, while for lower densities the present results provide a lower bound of dynamical correlations on $\operatorname{Im} \epsilon(k, \omega)$ outside the ph spectrum.

Simple metals provide the closest realizations of the jellium model in nature. Dynamical correlations as corrections to the RPA of the jellium model compete with band-structure effects in the RPA due to the weak pseudopotential, so that band-structure effects provide a natural measure for the relative importance of dynamical correlations. For Al we have evaluated band-structure contributions to $\operatorname{Im} \epsilon(\mathbf{k}, \omega)$ in second-order perturbation theory using an Ashcroft empty core potential. While for small $k$ band-structure effects are dominant, correlation effects increase with increasing $k$ in importance and finally prevail for $k \geqslant 1$.

We have applied the results to discuss various experiments. Plasmon damping at small $k$ is essentially caused by interband transitions and local-field effects, but close to $q_{c}$ the decay via ph-ph and ph-pl excitations makes an important contribution as we demonstrated for Al.

In recent years inelastic x-ray scattering has become a powerful tool to investigate the dynamical structure factor at large $k\left(k>q_{c}\right)$, and our results are applicable to the highfrequency tail, i.e., for $\omega>k^{2}+2 k$. Here we have demonstrated that at large $k \quad(k \geqslant 1)$ dynamical correlation effects in terms of multiple excitations are more important than band-structure effects. Single particle-hole excitations (interband transitions) are insufficient to explain the observed magnitude of the tail, while multiple excitations give the right order of magnitude. A particularly interesting aspect is the existence of the pl-pl peak. We found in one of the experimental spectra $(k=0.66)$ of Ref. 23 a small structure of roughly the appropriate position and magnitude. Further experimental investigations of the high-frequency tail would be very valuable.

Finally we investigated the effect of dynamical correla- tions on the optical properties of the alkali metals. Below the onset of interband transitions a strong deviation from the expected Drude behavior is observed. Multiple excitations mediated by the crystal potential could be responsible for this observation, as we have demonstrated for $\mathrm{K}$ and $\mathrm{Rb}$. The same effect is present in $\mathrm{Na}$, but an unexpectedly large pseudopotential coefficient $V_{\mathbf{G}_{110}}$ is required as for the magnitude of the interband transitions.

These applications show that dynamical correlations as defined by the diagrams in Fig. 1 can substantially influence measurable properties, and the magnitude of the correlation contribution is physically reasonable in all cases considered.

A final judgment of the quality of the approximation defined by the diagrams in Fig. 1 will be possible when their effect inside the ph spectrum is evaluated, and we plan to extend the calculation to $\operatorname{Im} \epsilon(k, \omega)$ to this case. Since the $f$ sum rule is satisfied in the RPA, additional excitations outside the ph spectrum must be accompanied by a reduction of the oscillator strength inside the ph spectrum. The third frequency moment sum rule depends explicitly on correlations and provides an additional way to check the quality of the approximation defined by the diagrams in Fig. 1. KramersKronig relations can then be applied to evaluate $\operatorname{Re} \epsilon(k, \omega)$.

The diagrams considered in this paper are a consistent subset in the sense that they lead to the correct $r_{s}$ dependence of the compressibility sum rule in the high-density limit (see discussion in Ref. 14). There are additional second-order exchange diagrams, which for $\omega \gg k^{2}+2 k$ can reduce $\operatorname{Im} \epsilon(k, \omega)$ by $50 \%,{ }^{7,19}$ but they do not contribute to the compressibility sum rule. These diagrams are considerably more difficult to evaluate exactly at arbitrary $(k, \omega)$, but it would be interesting to obtain some estimate. Higher-order HartreeFock diagrams could be included approximately in a form proposed by Geldart and Taylor. ${ }^{15}$ Their result for the large$k$ behavior of the static screening function was recently confirmed in part by Monte Carlo simulations. ${ }^{29}$

\section{ACKNOWLEDGMENTS}

We are grateful to A. Bringer, E. Eisenriegler, R. O. Jones, A. Liebsch, and H. Lustfeld for useful discussions and comments.

\section{APPENDIX: EVALUATION OF THE DIAGRAMS}

Here we outline the technical details used to evaluate the diagrams of Fig. 1, whose formal representation was given in Sec. II.

\section{Im $\epsilon_{A B C}(k, \omega)$ outside the particle-hole excitation spectrum}

The $m$ summation in $\pi_{A B}\left(k, \omega_{n}\right)$ and $\pi_{C}\left(k, \omega_{n}\right)$ (see Sec. II) is performed by again converting the sum into a contour integral (see Refs. 9 and 13). Finally the functions defined on the imaginary frequency axis are continued analytically into the upper complex $\omega$ plane in the limit $T \rightarrow 0$. Then, $\pi_{A B}(k, \omega) \equiv \pi_{A B}(k, \omega+i \delta) \quad$ and $\quad \pi_{C}(k, \omega) \equiv \pi_{C}(k, \omega+i \delta)$ consist of terms of the following form:

$$
H(\omega+i \delta) I(\omega+i \delta)
$$

with 


$$
\begin{aligned}
I(\omega+i \delta)= & \frac{-1}{\pi} \int_{-\infty}^{0} d x \operatorname{Im}[f(x+i \epsilon)] g(\omega-x+i \delta) \\
& +\frac{1}{\pi} \int_{-\infty}^{\omega} d x f(x+i \epsilon) \operatorname{Im}[g(\omega-x+i \delta)],
\end{aligned}
$$

where we have suppressed any wave vector dependency for the time being, and $\delta$ and $\epsilon$ are positive infinitesimals. $H(\omega+i \delta)$ depends on denominators of the form $[ \pm(\omega$ $\left.+i \delta)+\varepsilon_{\mathbf{p}}-\varepsilon_{\mathbf{p}+\mathbf{k}}\right]$ in zero, first, or second order. Thus $H(\omega+i \delta)=H(\omega)$ is real outside the ph spectrum, and the imaginary parts $\operatorname{Im} \pi_{A B}(k, \omega)$ and $\operatorname{Im} \pi_{C}(k, \omega)$ consist of terms of the form

$$
H(\omega) \frac{1}{\pi} \int_{0}^{\omega} d x \operatorname{Im}[f(x+i \epsilon)] \operatorname{Im}[g(\omega-x+i \delta)] .
$$

In the approximation defined by the above diagrams we have

$$
\operatorname{Im} \epsilon_{A B C}(k, \omega)=v(k) \operatorname{Im}\left[\pi_{A B}(k, \omega)+\pi_{C}(k, \omega)\right] .
$$

We apply the relationship

$$
U_{q}(x) \equiv \frac{v(q)}{\epsilon_{L}(q, x)}=v(q)-v^{2}(q) \frac{\pi_{0}(q, x)}{\epsilon_{L}(q, x)}
$$

in the expression of $\pi_{A B}(k, \omega)$ and observe that only the term $\sim v^{2}(q)$ contributes to $\operatorname{Im} \epsilon_{A B C}(k, \omega)$ outside the ph spectrum. The term $\sim v(q)$ represents the lowest order Hartree-Fock approximation ${ }^{17}$ and its imagimary part vanishes there. Furthermore, the term $F_{2}$ in $\pi_{A B}$ [Eq. (2.9)] does not contribute, because it is independent of $\omega-x$ and thus $\operatorname{Im}[g(\omega-x+i \delta)]=0$. Following the rules indicated above we finally have to evaluate

$$
\begin{aligned}
\operatorname{Im} \pi_{A B}(k, \omega)= & \int \frac{d^{3} q}{(2 \pi)^{3}} v(q)^{2} \int_{0}^{\omega} \frac{d x}{\pi}\left\{\operatorname{Im}\left[\frac{\pi_{0}(q, x+i \boldsymbol{\epsilon})}{\epsilon_{L}(q, x+i \epsilon)}\right] \int \frac{d^{3} p}{(2 \pi)^{2}} f\left(\varepsilon_{\mathbf{p}}\right) \delta\left(y+\varepsilon_{\mathbf{p}}-\varepsilon_{\mathbf{p}+\mathbf{k}+\mathbf{q}}\right)\right. \\
& \times \operatorname{Re}\left[\left(\frac{1}{\omega+\varepsilon_{\mathbf{p}}-\varepsilon_{\mathbf{p}+\mathbf{k}}}-\frac{1}{\omega+i \delta+\varepsilon_{\mathbf{p}+\mathbf{q}}-\varepsilon_{\mathbf{p}+\mathbf{k}+\mathbf{q}}}\right)^{2}\right]+\operatorname{Re}\left[\frac{\pi_{0}(q, x+i \boldsymbol{\epsilon})}{\epsilon_{L}(q, x+i \boldsymbol{\epsilon})}\right] \int \frac{d^{3} p}{(2 \pi)^{2}} f\left(\varepsilon_{\mathbf{p}}\right) \delta\left(y+\varepsilon_{\mathbf{p}}-\varepsilon_{\mathbf{p}+\mathbf{k}+\mathbf{q}}\right) \\
& \times \operatorname{Im}\left[\left(\frac{1}{\omega+\varepsilon_{\mathbf{p}}-\varepsilon_{\mathbf{p}+\mathbf{k}}}-\frac{1}{\omega+i \delta+\varepsilon_{\mathbf{p}+\mathbf{q}}-\varepsilon_{\mathbf{p}+\mathbf{k}+\mathbf{q}}}\right)^{2}\right]-\operatorname{Im}\left[\frac{\pi_{0}(q, x+i \boldsymbol{\epsilon})}{\epsilon_{L}(q, x+i \boldsymbol{\epsilon})}\right] \int \frac{d^{3} p}{(2 \pi)^{2}} f\left(\varepsilon_{\mathbf{p}}\right) \delta\left(y-\varepsilon_{\mathbf{p}}+\varepsilon_{\mathbf{p}+\mathbf{k}+\mathbf{q})}\right. \\
& \times \operatorname{Re}\left[\left(\frac{-1}{\omega-\varepsilon_{\mathbf{p}}+\varepsilon_{\mathbf{p}+\mathbf{k}}}-\frac{-1}{\omega+i \delta-\varepsilon_{\mathbf{p}+\mathbf{q}}+\varepsilon_{\mathbf{p}+\mathbf{k}+\mathbf{q}}}\right)^{2}\right]-\operatorname{Re}\left[\frac{\pi_{0}(q, x+i \boldsymbol{\epsilon})}{\epsilon_{L}(q, x+i \boldsymbol{\epsilon})}\right] \int \frac{d^{3} p}{(2 \pi)^{2}} f\left(\varepsilon_{\mathbf{p}}\right) \delta\left(y-\varepsilon_{\mathbf{p}}+\varepsilon_{\mathbf{p}+\mathbf{k}+\mathbf{q})}\right. \\
& \left.\times \operatorname{Im}\left[\left(\frac{-1}{\omega-\varepsilon_{\mathbf{p}}+\varepsilon_{\mathbf{p}+\mathbf{k}}}-\frac{-1}{\omega+i \delta-\varepsilon_{\mathbf{p}+\mathbf{q}}+\varepsilon_{\mathbf{p}+\mathbf{k}+\mathbf{q}}}\right)^{2}\right]\right\}
\end{aligned}
$$

with $y=\omega-x$. In obtaining this result we made use of the $y$-dependent $\delta$ functions to rewrite the denominators that depend on the internal frequency variable $x$.

The $\mathbf{p}$ integrals can be represented in terms of elementary functions. In the remaining $\mathbf{q}$ integrals the integrand is independent of azimuthal angle when $\mathbf{k}$ is used as polar axis. Thus we are left to perform three integrations $\left(q_{\perp}, q_{\|}, x\right)$ numerically. $\operatorname{Im} \pi_{C}(k, \omega)$ has a similar structure, as will be shown below.

\section{2. $p$ integrals}

Before we consider the expression for $\operatorname{Im} \pi_{C}(k, \omega)$ outside the ph spectrum it is convenient to list the $\mathbf{p}$ integrals that occur in $\operatorname{Im} \pi_{A B}(k, \omega)$ and $\operatorname{Im} \pi_{C}(k, \omega)$. We show that they have a similar structure and can be represented in terms of elementary functions.

Outside the ph spectrum

$$
\pm \omega+\varepsilon_{\mathbf{p}}-\varepsilon_{\mathbf{p}+\mathbf{k}} \neq 0
$$

and all the $\mathbf{p}$ integrals that one must solve to obtain $\operatorname{Im} \pi_{A B}(k, \omega)$ and $\operatorname{Im} \pi_{C}(k, \omega)$ can be related to eight integrals which we list below:

$$
\begin{aligned}
h_{1}(\mathbf{q}, y+i \delta ; \mathbf{k}, \omega)= & 2 \int \frac{d^{3} p}{(2 \pi)^{3}} f\left(\varepsilon_{\mathbf{p}}\right) \frac{1}{\omega+\varepsilon_{\mathbf{p}}-\varepsilon_{\mathbf{p}+\mathbf{k}}} \\
& \times \frac{1}{y+i \delta+\varepsilon_{\mathbf{p}}-\varepsilon_{\mathbf{p}+\mathbf{k}+\mathbf{q}}} \\
h_{2}\left(\mathbf{q}, x+i \epsilon^{\prime} ; \mathbf{k}, \omega\right)= & 2 \int \frac{d^{3} p}{(2 \pi)^{3}} f\left(\varepsilon_{\mathbf{p}}\right) \frac{1}{-\omega+\varepsilon_{\mathbf{p}}-\varepsilon_{\mathbf{p}+\mathbf{k}}} \\
& \times \frac{1}{-\left(x+i \epsilon^{\prime}\right)+\varepsilon_{\mathbf{p}}-\varepsilon_{\mathbf{p}-\mathbf{q}}}
\end{aligned}
$$




$$
\begin{aligned}
h_{3}\left(\mathbf{q}, x \pm i \boldsymbol{\epsilon}^{\prime} ; \mathbf{k}, \omega \pm i \epsilon\right)=2 \int & \frac{d^{3} p}{(2 \pi)^{3}} f\left(\varepsilon_{\mathbf{p}}\right) \\
& \times \frac{1}{-(\omega \pm i \boldsymbol{\epsilon})+\varepsilon_{\mathbf{p}+\mathbf{q}}-\varepsilon_{\mathbf{p}+\mathbf{k}+\mathbf{q}}} \\
& \times \frac{1}{x \pm i \boldsymbol{\epsilon}^{\prime}+\varepsilon_{\mathbf{p}}-\varepsilon_{\mathbf{p}+\mathbf{q}}}, \quad(\mathrm{A} 10) \\
h_{4}(\mathbf{q}, y \pm i \delta ; \mathbf{k}, \omega \pm i \boldsymbol{\epsilon})=- & 2 \int \frac{d^{3} p}{(2 \pi)^{3}} f\left(\varepsilon_{\mathbf{p}}\right) \\
\times & \frac{1}{-(\omega \pm i \boldsymbol{\epsilon})+\varepsilon_{\mathbf{p}+\mathbf{q}}-\varepsilon_{\mathbf{p}+\mathbf{k}+\mathbf{q}}} \\
& \times \frac{1}{-(y \pm i \delta)+\varepsilon_{\mathbf{p}}-\varepsilon_{\mathbf{p}+\mathbf{k}+\mathbf{q}}} .
\end{aligned}
$$

(A11)

The functions $h_{5}, h_{6}, h_{7}$, and $h_{8}$ follow from $h_{1}, h_{2}, h_{3}$, and $h_{4}$ by replacing $\omega$ by $-\omega, \omega \pm i \epsilon$ by $-(\omega \pm i \epsilon), \quad x$ $\pm i \epsilon^{\prime}$ by $-\left(x \pm i \epsilon^{\prime}\right)$, and $y \pm i \delta$ by $-(y \pm i \delta)$. While all the functions $h_{i}, \quad i=1, \ldots, 8$ enter the expression for $\operatorname{Im} \pi_{C}(k, \omega)$, the $\mathbf{p}$ integrals in $\operatorname{Im} \pi_{A B}(k, \omega)$ are related to $h_{1}, h_{4}, h_{5}$, and $h_{8}$, and their first derivatives with respect to $\omega$ keeping $y$ fixed.

We now indicate the evaluation of the $h_{i}, \quad i=1, \ldots, 8$. If we write the integrals in cylindrical coordinates choosing $\mathbf{k}$ as polar axis, the structures of the $h_{i}$ are identical. With a common prefactor $A=k_{F}^{3} / \varepsilon_{F}^{2}$ all variables and parameters are dimensionless and, except for the minus sign in $h_{4}$ and $h_{8}$, the integrals have the same form, namely

$$
\begin{aligned}
h= & 2 A \int_{-1}^{+1} d p_{\|} \frac{1}{\gamma \pm i \epsilon-p_{\|}} \int_{0}^{\sqrt{1-p_{\|}^{2}}} d p_{\perp} p_{\perp} \\
& \times \int_{0}^{2 \pi} d \varphi \frac{1}{\phi\left(p_{\|}\right) \pm i \delta-2 p_{\perp} q_{\perp} \cos \varphi} \\
= & A[\text { hrr }+ \text { hii } i \text { ihri } i \text { hir }] .
\end{aligned}
$$

The $\varphi$ and $p_{\perp}$ integrations are easily performed and we have $\left(x \equiv p_{\|}\right)$

$$
\begin{gathered}
h r r=-\frac{\pi}{2 k q_{\perp}^{2}} \mathcal{P} \int_{-1}^{+1} \frac{d x}{x-\gamma}\{\phi(x) \\
-\sqrt{P(x)} \theta[P(x)] \operatorname{sgn}[\phi(x)]\}, \\
h i i=\frac{\pi^{2}}{2 k q_{\perp}^{2}} \int_{-1}^{+1} d x \delta(x-\gamma) \sqrt{-P(x)} \theta[-P(x)] \\
=\frac{\pi^{2}}{2 k q_{\perp}^{2}} \sqrt{-P(\gamma)} \Theta[-P(\gamma)] \Theta\left(1-\gamma^{2}\right), \\
h r i=-\frac{\pi}{2 k q_{\perp}^{2}} \mathcal{P} \int_{-1}^{+1} \frac{d x}{x-\gamma} \sqrt{-P(x)} \theta[-P(x)],
\end{gathered}
$$

$$
\begin{aligned}
\text { hir }= & \frac{\pi^{2}}{2 k q_{\perp}^{2}} \int_{-1}^{+1} d x \delta(x-\gamma)\{\phi(x) \\
& -\sqrt{P(x)} \theta[P(x)] \operatorname{sgn}[\phi(x)]\} \\
= & \frac{\pi^{2}}{2 k q_{\perp}^{2}}\{\phi(\gamma)-\sqrt{P(\gamma)} \Theta[P(\gamma)] \operatorname{sgn}[\phi(\gamma)]\} \Theta\left(1-\gamma^{2}\right),
\end{aligned}
$$

where

$$
\phi(x)=t-u x
$$

$t$ and $u$ are real and

$$
P(x)=\phi(x)^{2}-4 q_{\perp}^{2}\left(1-x^{2}\right) \equiv a x^{2}+2 b x+c
$$

is a quadratic polynomial, $\Theta(x)$ is the unit step function, and $\mathcal{P}$ denotes the principal value. The principal value integrals can also be represented in terms of elementary functions. We define

$$
h r r=\frac{\pi}{2 k q_{\perp}^{2}}\left[J_{1}+J_{2}\right]
$$

with

$$
J_{1}=-\mathcal{P} \int_{-1}^{+1} d x \frac{\phi(x)}{x-\gamma}=2 u+(t-\gamma u) \ln \left|\frac{\gamma+1}{\gamma-1}\right|
$$

and

$$
J_{2}=\mathcal{P} \int_{-1}^{+1} \frac{d x}{x-\gamma} \sqrt{P(x)} \theta[P(x)] \operatorname{sgn}[\phi(x)] .
$$

To perform the $x$ integration we use the identity

$$
\frac{\sqrt{P(x)}}{x-\gamma}=\frac{\left[P^{\prime}(x)+P^{\prime}(\gamma)\right]}{2 \sqrt{P(x)}}+\frac{P(\gamma)}{\sqrt{P(x)}} \frac{1}{(x-\gamma)},
$$

where $P^{\prime}(x)=d P(x) / d x$. Thus we have to solve three different principal value integrals:

$$
J_{2}=J_{21}+J_{22}+J_{23} \text {. }
$$

To evaluate $J_{2}$ several cases must be considered depending on the roots of $P(x)$.

Case A:

$$
b^{2}-a c<0
$$

Then $P(x)$ has no real roots, $P(x)>0, \theta[P(x)]=1$, and $\phi(x)$ does not change sign on $(-1,1)$ and we obtain

$$
J_{21}=\operatorname{sgn}[\phi(1)] \int_{-1}^{+1} d x \frac{P^{\prime}(x)}{\sqrt{P(x)}}=\phi(1)-\phi(-1),
$$




$$
\begin{aligned}
J_{22}= & \operatorname{sgn}[\phi(1)] \frac{P^{\prime}(\gamma)}{2} \int_{-1}^{+1} \frac{d x}{\sqrt{P(x)}} \\
= & \operatorname{sgn}[\phi(1)] \frac{P^{\prime}(\gamma)}{2 \sqrt{a}} \\
& \times \ln \left|\frac{P^{\prime}(1)+2 \sqrt{a}|\phi(1)|}{P^{\prime}(-1)+2 \sqrt{a} \mid \phi(-1)}\right|, \\
J_{23}= & \operatorname{sgn}[\phi(1)] P(\gamma) \mathcal{P} \int_{-1}^{+1} \frac{d x}{\sqrt{P(x)}} \frac{1}{x-\gamma} \\
= & \operatorname{sgn}[\phi(1)] \sqrt{P(\gamma)}\left\{\ln \left|\frac{1-\gamma}{1+\gamma}\right|-\ln \left|\frac{f(1, \gamma)}{f(-1, \gamma)}\right|\right\},
\end{aligned}
$$

with

$$
f(x, \gamma)=0.5(x-\gamma) P^{\prime}(\gamma)+P(\gamma)+\sqrt{P(x) P(\gamma)}
$$

Case B:

$$
b^{2}-a c>0 \text {. }
$$

Then $P(x)$ has two real roots $x_{1,2}=-b / a \mp \sqrt{b^{2}-a c}$ / $a \epsilon[-1,1]$, since $a>0$ and because of the form of $P(x)$ [see Eq. (A19)] we find

$$
J_{21}=\left[\int_{-1}^{x_{1}}+\int_{x_{2}}^{1}\right] d x \frac{P^{\prime}(x)}{2 \sqrt{P(x)}} \operatorname{sgn}[\phi(x)]=\phi(1)-\phi(-1),
$$

$$
\begin{aligned}
J_{22}= & \frac{P^{\prime}(\gamma)}{2}\left[\int_{-1}^{x_{1}}+\int_{x_{2}}^{1}\right] d x \frac{\operatorname{sgn}[\phi(x)]}{\sqrt{P(x)}} \\
= & \frac{P^{\prime}(\gamma)}{2}\left\{\ln \left|\frac{P^{\prime}\left(x_{1}\right)}{P^{\prime}(-1)+2 \sqrt{a}|\phi(-1)|}\right| \operatorname{sgn}[\phi(-1)]\right. \\
& \left.-\ln \left|\frac{P^{\prime}\left(x_{2}\right)}{P^{\prime}(1)+2 \sqrt{a}|\phi(1)|}\right| \operatorname{sgn}[\phi(1)]\right\} .
\end{aligned}
$$

To calculate $J_{23}$ we distinguish two cases:

(i) $P(\gamma)>0$;

$$
\begin{aligned}
J_{23}= & P(\gamma) \mathcal{P}\left[\int_{-1}^{x_{1}}+\int_{x_{2}}^{1}\right] d x \frac{1}{\sqrt{P(x)}} \frac{1}{x-\gamma} \operatorname{sgn}[\phi(x)] \\
= & \sqrt{P(\gamma)}\left\{\left(\ln \left|\frac{x_{1}-\gamma}{1+\gamma}\right|-\ln \left|\frac{f\left(x_{1}, \gamma\right)}{f(-1, \gamma)}\right|\right) \operatorname{sgn}[\phi(-1)]\right. \\
& \left.+\left(\ln \left|\frac{1-\gamma}{x_{2}+\gamma}\right|-\ln \left|\frac{f(1, \gamma)}{f\left(x_{2}, \gamma\right)}\right|\right) \operatorname{sgn}[\phi(1)]\right\} .
\end{aligned}
$$

(ii) $P(\gamma)<0$;

$$
\begin{aligned}
J_{23}= & P(\gamma) \mathcal{P}\left[\int_{-1}^{x_{1}}+\int_{x_{2}}^{1}\right] d x \frac{1}{\sqrt{P(x)}} \frac{1}{x-\gamma} \operatorname{sgn}[\phi(x)] \\
= & -\sqrt{-P(\gamma)} \operatorname{sgn}[\phi(-1)]\left(\left\{\arcsin \left[g\left(x_{1}, \gamma\right)\right]\right.\right. \\
& -\arcsin [g(-1, \gamma)]\}+\{\arcsin [g(1, \gamma)] \\
& \left.\left.-\arcsin \left[g\left(x_{2}, \gamma\right)\right]\right\} \operatorname{sgn}[\phi(1)]\right),
\end{aligned}
$$

with

$$
g(x, \gamma)=\frac{0.5(x-\gamma) P^{\prime}(\gamma)+P(\gamma)}{\sqrt{\left(b^{2}-a c\right)}|x-\gamma|}
$$

Case C:

$$
b^{2}-a c=0 .
$$

Then $x_{1,2}=x_{0}=-b / a$ with $a>0, \quad P(x)=a\left(x-x_{0}\right)^{2}$ with $\left|x_{0}\right|<1, \quad \phi(x)$ does not change sign in $[-1,1]$ and we obtain

$$
\begin{aligned}
J_{2} & =\mathcal{P} \int_{-1}^{1} d x \frac{\operatorname{sgn}[\phi(x)] \sqrt{P(x)}}{x-\gamma} \\
& =\sqrt{a}\left\{\left(\gamma-x_{0}\right) \ln \left|\frac{1-\gamma^{2}}{\left(x_{0}-\gamma\right)^{2}}\right|-2 x_{0}\right\} \operatorname{sgn}[\phi(1)] .
\end{aligned}
$$

This completes the integrations in $h r r$ and we now turn to hri. Similar manipulations result in

$$
h r i=\frac{\pi^{2}}{2 k q_{\perp}^{2}}\left\{P^{\prime}(\gamma) / 2 \sqrt{a}+\sqrt{P(\gamma)} d(\gamma)\right\} \Theta\left(b^{2}-a c\right),
$$

where

$$
\begin{aligned}
d(\gamma) & =1, \quad \gamma<x_{1}, \\
& =0, \quad \gamma \epsilon\left[x_{1}, x_{2}\right] \\
& =-1, \quad \gamma>x_{2} .
\end{aligned}
$$

It is now straightforward to obtain the derivatives of the appropriate $h$ functions required for the evaluation of $\operatorname{Im} \pi_{A B}(k, \omega)$.

\section{Im $\pi_{A B}(k, \omega)$ in terms of the $h_{i}$ functions}

Here we show that the $\mathbf{p}$ integrals in $\operatorname{Im} \pi_{A B}(k, \omega)$ can be expressed in terms of the functions $h_{i}, i=1,4,5,8$ and their derivatives with respect to $\omega$ with $y$ fixed. 


$$
\begin{aligned}
\operatorname{Im} \pi_{A B}(k, \omega)= & \int \frac{d^{3} q}{(2 \pi)^{3}} v(q) \int_{0}^{\omega} \frac{d x}{\pi}\left\{\operatorname { I m } ( \frac { - 1 } { \boldsymbol { \epsilon } _ { L } ( q , x + i \epsilon ) } ) \left[\left(\frac{\partial}{\partial \omega}+\frac{m_{e}}{\hbar^{2} \mathbf{k} \cdot \mathbf{q}}\right) \operatorname{Im}\left[h_{1}(y+i \delta, \omega)\right]\right.\right. \\
& \left.+\left(\frac{\partial}{\partial \omega}-\frac{m_{e}}{\hbar^{2} \mathbf{k} \cdot \mathbf{q}}\right) H_{8}(\mathbf{q}, y ; \mathbf{k}, \omega)\right]+\operatorname{Re}\left(1-\frac{1}{\epsilon_{L}(q, x+i \boldsymbol{\epsilon})}\right)\left(\frac{\partial}{\partial \omega}+\frac{m_{e}}{\hbar^{2} \mathbf{k} \cdot \mathbf{q}}\right) \\
& \times G_{8}(\mathbf{q}, y ; \mathbf{k}, \omega)-\operatorname{Im}\left(\frac{-1}{\epsilon_{L}(q, x+i \epsilon)}\right)\left[\left(\frac{\partial}{\partial \omega}-\frac{m_{e}}{\hbar^{2} \mathbf{k} \cdot \mathbf{q}}\right) \operatorname{Im}\left[h_{5}(y+i \delta, \omega)\right]\right. \\
& \left.\left.-\left(\frac{\partial}{\partial \omega}+\frac{m_{e}}{\hbar^{2} \mathbf{k} \cdot \mathbf{q}}\right) H_{4}(\mathbf{q}, y ; \mathbf{k}, \omega)\right]+\operatorname{Re}\left(1-\frac{1}{\epsilon_{L}(q, x+i \boldsymbol{\epsilon})}\right)\left(\frac{\partial}{\partial \omega}-\frac{m_{e}}{\hbar^{2} \mathbf{k} \cdot \mathbf{q}}\right) G_{4}(\mathbf{q}, y ; \mathbf{k}, \omega)\right\},
\end{aligned}
$$

where

$$
\begin{aligned}
H_{4}(\mathbf{q}, y ; \mathbf{k}, \omega)= & (1 / 4 i)\left\{h_{4}(y+i \delta, \omega+i \epsilon)-h_{4}(y-i \delta, \omega+i \epsilon)\right. \\
& \left.+h_{4}(y+i \delta, \omega-i \epsilon)-h_{4}(y-i \delta, \omega-i \epsilon)\right\},
\end{aligned}
$$

$H_{8}(\mathbf{q}, y ; \mathbf{k}, \omega)=(1 / 4 i)\left\{h_{8}(y+i \delta, \omega+i \epsilon)-h_{8}(y-i \delta, \omega+i \epsilon)\right.$

$$
\left.+h_{8}(y+i \delta, \omega-i \epsilon)-h_{8}(y-i \delta, \omega-i \epsilon)\right\},
$$

$$
\begin{aligned}
G_{4}(\mathbf{q}, y ; \mathbf{k}, \omega)= & (-1 / 4)\left\{h_{4}(y+i \delta, \omega+i \epsilon)\right. \\
& -h_{4}(y-i \delta, \omega+i \epsilon)-h_{4}(y+i \delta, \omega-i \epsilon) \\
& \left.+h_{4}(y-i \delta, \omega-i \epsilon)\right\}
\end{aligned}
$$

$$
\begin{aligned}
G_{8}(\mathbf{q}, y ; \mathbf{k}, \omega)= & (-1 / 4)\left\{h_{8}(y+i \delta, \omega+i \epsilon)\right. \\
& -h_{8}(y-i \delta, \omega+i \epsilon)-h_{8}(y+i \delta, \omega-i \epsilon) \\
& \left.+h_{8}(y-i \delta, \omega-i \epsilon)\right\}
\end{aligned}
$$

and we made use of the relations

$$
\operatorname{Re}\left[\frac{1}{x+i \epsilon}\right]=\frac{1}{2}\left(\frac{1}{x+i \epsilon}+\frac{1}{x-i \epsilon}\right)
$$

and

$$
\operatorname{Im}\left[\frac{1}{x+i \epsilon}\right]=\frac{1}{2 i}\left(\frac{1}{x+i \epsilon}-\frac{1}{x-i \epsilon}\right) .
$$

\section{4. $\operatorname{Im} \pi_{C}(k, \omega)$ in terms of the $h_{i}$ functions}

To evaluate $\operatorname{Im} \pi_{C}(k, \omega)$ according to the rules indicated above we need to order the products appropriately. This will be done in several steps. Suppressing the wave-vector dependencies as far as possible we write

$$
\begin{aligned}
F_{3}\left(\omega_{n},-\alpha_{m}\right)= & h_{1}\left(\omega_{n}-\alpha_{m}, \omega_{n}\right)+h_{2}\left(\alpha_{m}, \omega_{n}\right) \\
& +2 \sum_{\mathbf{p}} f\left(\varepsilon_{\mathbf{p}}\right) a_{1}\left(\omega_{n}-\alpha_{m}, \omega_{n}, \mathbf{p}\right) b_{1}\left(\alpha_{m}, \mathbf{p}\right)
\end{aligned}
$$

and

$$
\begin{aligned}
F_{3}\left(-\omega_{n}, \alpha_{m}\right)= & h_{5}\left(\omega_{n}-\alpha_{m}, \omega_{n}\right)+h_{6}\left(\alpha_{m}, \omega_{n}\right) \\
& +2 \sum_{\mathbf{p}} f\left(\varepsilon_{\mathbf{p}}\right) a_{2}\left(\omega_{n}-\alpha_{m}, \omega_{n}, \mathbf{p}\right) b_{2}\left(\alpha_{m}, \mathbf{p}\right),
\end{aligned}
$$

where $h_{1}, h_{2}, h_{5}$, and $h_{6}$ are the functions defined above, with the replacement of $\omega$ by $\omega_{n}, x+i \epsilon^{\prime}$ by $\alpha_{m}$, and $y$ $+i \delta$ by $\omega_{n}-\alpha_{m}$, and

$$
\begin{gathered}
a_{1}\left(\omega_{n}-\alpha_{m}, \mathbf{p}\right)=1 /\left[-\left(\omega_{n}-\alpha_{m}\right)+\varepsilon_{\mathbf{p}}-\varepsilon_{\mathbf{p}+\mathbf{k}+\mathbf{q}}\right], \\
a_{2}\left(\omega_{n}-\alpha_{m}, \mathbf{p}\right)=1 /\left[\omega_{n}-\alpha_{m}+\varepsilon_{\mathbf{p}}-\varepsilon_{\mathbf{p}+\mathbf{k}+\mathbf{q}}\right], \\
b_{1}\left(\alpha_{m}, \mathbf{p}\right)=1 /\left[\alpha_{m}+\varepsilon_{\mathbf{p}}-\varepsilon_{\mathbf{p}+\mathbf{q}}\right]
\end{gathered}
$$

and

$$
b_{2}\left(\alpha_{m}, \mathbf{p}\right)=1 /\left[-\alpha_{m}+\varepsilon_{\mathbf{p}}-\varepsilon_{\mathbf{p}+\mathbf{q}}\right] .
$$

We write

$$
\pi_{C}\left(k, \omega_{n}\right)=\sum_{i=1}^{3} \pi_{C i}\left(k, \omega_{n}\right),
$$

where

$$
\begin{aligned}
\pi_{C 1}\left(k, \omega_{n}\right)= & \int \frac{d^{3} q}{(2 \pi)^{3}} \frac{1}{\beta} \sum_{\alpha_{m}} U_{\mathbf{q}}\left(\alpha_{m}\right) U_{\mathbf{q}+\mathbf{k}}\left(\omega_{n}-\alpha_{m}\right) \\
& \times h_{1}\left(\omega_{n}-\alpha_{m}, \omega_{n}\right)\left[F_{C}\left(\omega_{n},-\alpha_{m}\right)\right. \\
& \left.+F_{3}\left(-\omega_{n}, \alpha_{m}\right)\right],
\end{aligned}
$$




$$
\pi_{C 2}\left(k, \omega_{n}\right)=\int \frac{d^{3} q}{(2 \pi)^{3}} \frac{1}{\beta} \sum_{\alpha_{m}} U_{\mathbf{q}}\left(\alpha_{m}\right) U_{\mathbf{q}+\mathbf{k}}\left(\omega_{n}-\alpha_{m}\right) h_{2}\left(\alpha_{m}, \omega_{n}\right)\left[F_{3}\left(\omega_{n},-\alpha_{m}\right)+F_{3}\left(-\omega_{n}, \alpha_{m}\right)\right]
$$

and

$$
\begin{aligned}
\pi_{C 3}\left(k, \omega_{n}\right)= & \int \frac{d^{3} q}{(2 \pi)^{3}} \frac{1}{\beta} \sum_{\alpha_{m}} U_{\mathbf{q}}\left(\alpha_{m}\right) U_{\mathbf{q}+\mathbf{k}}\left(\omega_{n}-\alpha_{m}\right) 2 \sum_{\mathbf{p}} f\left(\varepsilon_{\mathbf{p}}\right) a_{1} \\
& \times\left(\omega_{n}-\alpha_{m}, \mathbf{p}\right) b_{1}\left(\alpha_{m}, \mathbf{p}\right)\left[F_{3}\left(\omega_{n},-\alpha_{m}\right)+F_{3}\left(-\omega_{n}, \alpha_{m}\right)\right] .
\end{aligned}
$$

When the $\alpha_{m}$ summation has been performed by contour integration and the result is continued into the upper complex frequency plane, we have $(y=\omega-x)$

$$
\begin{aligned}
& \operatorname{Im} \pi_{C 1}(k, \omega+i \delta)=\int_{0}^{\omega} \frac{d x}{\pi} \int \frac{d^{3} q}{(2 \pi)^{3}}\left(\operatorname{Im}\left[U_{\mathbf{q}}(x+i \epsilon)\right] \operatorname{Im}\left\{U_{\mathbf{q}+\mathbf{k}}(y+i \delta) h_{1}(y+i \delta, \omega)\left[h_{1}(y+i \delta, \omega)+h_{5}(y+i \delta, \omega)\right]\right\}\right. \\
& +\operatorname{Im}\left[U_{\mathbf{q}+\mathbf{k}}(y+i \delta) h_{1}(y+i \delta, \omega)\right] \operatorname{Im}\left\{U_{\mathbf{q}}(x+i \epsilon)\left[h_{2}(x+i \epsilon, \omega)+h_{6}(x+i \epsilon, \omega)\right]\right\} \\
& +2 \sum_{\mathbf{p}} f\left(\varepsilon_{\mathbf{p}}\right) \operatorname{Im}\left[U_{\mathbf{q}+\mathbf{k}}(y+i \delta) h_{1}(y+i \delta, \omega) a_{1}(y+i \delta)\right] \operatorname{Im}\left[U_{\mathbf{q}}(x+i \epsilon) b_{1}(x+i \epsilon)\right] \\
& \left.+2 \sum_{\mathbf{p}} f\left(\varepsilon_{\mathbf{p}}\right) \operatorname{Im}\left[U_{\mathbf{q}+\mathbf{k}}(y+i \delta) h_{1}(y+i \delta, \omega) a_{2}(y+i \delta)\right] \operatorname{Im}\left[U_{\mathbf{q}}(x+i \boldsymbol{\epsilon}) b_{2}(x+i \boldsymbol{\epsilon})\right]\right), \\
& \operatorname{Im} \pi_{C 2}(k, \omega+i \delta)=\int_{0}^{\omega} \frac{d x}{\pi} \int \frac{d^{3} q}{(2 \pi)^{3}}\left(\operatorname{Im}\left\{U_{\mathbf{q}+\mathbf{k}}(y+i \delta)\right] \operatorname{Im}\left[U_{\mathbf{q}}(x+i \boldsymbol{\epsilon}) h_{2}(x+i \boldsymbol{\epsilon}, \omega)\left[h_{2}(x+i \boldsymbol{\epsilon}, \omega)+h_{6}(x+i \boldsymbol{\epsilon}, \omega)\right]\right\}\right. \\
& +\operatorname{Im}\left[U_{\mathbf{q}}(x+i \epsilon) h_{2}(x+i \epsilon, \omega)\right] \operatorname{Im}\left\{U_{\mathbf{q}+\mathbf{k}}(y+i \delta)\left[h_{1}(y+i \delta, \omega)+h_{5}(y+i \delta, \omega)\right]\right\} \\
& +2 \sum_{\mathbf{p}} f\left(\varepsilon_{\mathbf{p}}\right) \operatorname{Im}\left[U_{\mathbf{q}}(x+i \boldsymbol{\epsilon}) h_{2}(x+i \boldsymbol{\epsilon}, \omega) b_{1}(x+i \boldsymbol{\epsilon})\right] \operatorname{Im}\left[U_{\mathbf{q}+\mathbf{k}}(y+i \delta) a_{1}(y+i \delta, \mathbf{p})\right] \\
& \left.+2 \sum_{\mathbf{p}} f\left(\varepsilon_{\mathbf{p}}\right) \operatorname{Im}\left[U_{\mathbf{q}}(x+i \epsilon) h_{2}(x+i \epsilon, \omega) b_{2}(x+i \boldsymbol{\epsilon})\right] \operatorname{Im}\left[U_{\mathbf{q}+\mathbf{k}}(y+i \delta,) a_{2}(y+i \delta, \mathbf{p})\right]\right),
\end{aligned}
$$

and

$$
\begin{aligned}
\operatorname{Im} \pi_{C 3}(k, \omega+i \delta)= & \int_{0}^{\omega} \frac{d x}{\pi} \int \frac{d^{3} q}{(2 \pi)^{3}}\left(2 \sum_{\mathbf{p}} f\left(\varepsilon_{\mathbf{p}}\right) \operatorname{Im}\left\{U_{\mathbf{q}+\mathbf{k}}(y+i \delta) a_{1}(y+i \delta, \mathbf{p})\left[h_{1}(y+i \delta, \omega)+h_{5}(y+i \delta, \omega)\right]\right\}\right. \\
& \times \operatorname{Im}\left[U_{\mathbf{q}}(x+i \epsilon) b_{1}(x+i \epsilon, \mathbf{p})\right]+2 \sum_{\mathbf{p}} f\left(\varepsilon_{\mathbf{p}}\right) \operatorname{Im}\left\{U _ { \mathbf { q } } ( x + i \boldsymbol { \epsilon } ) b _ { 1 } ( x + i \epsilon , \mathbf { p } ) \left[h_{2}(x+i \epsilon, \omega)\right.\right. \\
& \left.\left.+h_{6}(x+i \epsilon, \omega)\right]\right\} \operatorname{Im}\left[U_{\mathbf{q}+\mathbf{k}}(y+i \delta) a_{1}(y+i \delta, \mathbf{p})\right]+4 \sum_{\mathbf{p}, \mathbf{p}^{\prime}} f\left(\varepsilon_{\mathbf{p}}\right) f\left(\varepsilon_{\mathbf{p}^{\prime}}\right) \\
& \times \operatorname{Im}\left[U_{\mathbf{q}+\mathbf{k}}(y+i \delta) a_{1}(y+i \delta, \mathbf{p}) a_{1}\left(y+i \delta, \mathbf{p}^{\prime}\right)\right] \operatorname{Im}\left[U_{\mathbf{q}}(x+i \epsilon) b_{1}(x+i \epsilon, \mathbf{p}) b_{1}\left(x+i \epsilon, \mathbf{p}^{\prime}\right)\right] \\
& +4 \sum_{\mathbf{p}, \mathbf{p}^{\prime}} f\left(\varepsilon_{\mathbf{p}}\right) f\left(\varepsilon_{\mathbf{p}^{\prime}}\right) \operatorname{Im}\left[U_{\mathbf{q}+\mathbf{k}}(y+i \delta) a_{1}(y+i \delta, \mathbf{p}) a_{2}\left(y+i \delta, \mathbf{p}^{\prime}\right)\right] \\
& \left.\times \operatorname{Im}\left[U_{\mathbf{q}}(x+i \epsilon) b_{1}(x+i \epsilon, \mathbf{p}) b_{2}\left(x+i \epsilon, \mathbf{p}^{\prime}\right)\right]\right)
\end{aligned}
$$


In the expressions for $\operatorname{Im} \pi_{C i}(k, \omega)$ the remaining $\mathbf{p}$ and $\mathbf{p}^{\prime}$ integrals involving the functions $a_{1}, a_{2}, b_{1}$, and $b_{2}$ can be expressed in terms of the functions $h_{3}, h_{4}, h_{7}$, and $h_{8}$ as is shown below:

$$
\begin{array}{rl}
2 \sum_{\mathbf{p}} & f\left(\varepsilon_{\mathbf{p}}\right) \operatorname{Re}\left[a_{1}(y+i \delta, \mathbf{p})\right] \operatorname{Im}\left[b_{1}\left(x+i \epsilon^{\prime}, \mathbf{p}\right)\right] \\
= & (1 / 4 i)\left\{h_{3}\left(x+i \epsilon^{\prime}, \omega+i \epsilon\right)-h_{3}\left(x-i \epsilon^{\prime}, \omega+i \epsilon\right)\right. \\
& \left.+h_{3}\left(x+i \epsilon^{\prime}, \omega-i \epsilon\right)-h_{3}\left(x-i \epsilon^{\prime}, \omega-i \epsilon\right)\right\},
\end{array}
$$

$$
\begin{array}{rl}
2 \sum_{\mathbf{p}} & f\left(\varepsilon_{\mathbf{p}}\right) \operatorname{Re}\left[a_{2}(y+i \delta, \mathbf{p})\right] \operatorname{Im}\left[b_{2}\left(x+i \epsilon^{\prime}, \mathbf{p}\right)\right] \\
= & (1 / 4 i)\left\{h_{5}\left(x+i \epsilon^{\prime}, \omega+i \epsilon\right)-h_{5}\left(x-i \epsilon^{\prime}, \omega+i \epsilon\right)\right. \\
& \left.+h_{5}\left(x+i \epsilon^{\prime}, \omega-i \epsilon\right)-h_{5}\left(x-i \epsilon^{\prime}, \omega-i \epsilon\right)\right\},
\end{array}
$$

$$
\begin{aligned}
2 \sum_{\mathbf{p}} f & \left(\varepsilon_{\mathbf{p}}\right) \operatorname{Re}\left[a_{1}(y+i \delta, \mathbf{p})\right] \operatorname{Re}\left[b_{1}\left(x+i \boldsymbol{\epsilon}^{\prime}, \mathbf{p}\right)\right] \\
= & (1 / 4)\left\{h_{3}\left(x+i \epsilon^{\prime}, \omega+i \boldsymbol{\epsilon}\right)+h_{3}\left(x-i \epsilon^{\prime}, \omega+i \epsilon\right)\right. \\
& +h_{3}\left(x+i \epsilon^{\prime}, \omega-i \epsilon\right)+h_{3}\left(x-i \epsilon^{\prime}, \omega-i \epsilon\right) \\
& +h_{4}(y+i \delta, \omega+i \epsilon)+h_{4}(y-i \delta, \omega+i \epsilon) \\
& \left.+h_{4}(y+i \delta, \omega-i \epsilon)+h_{4}(y-i \delta, \omega-i \epsilon)\right\},
\end{aligned}
$$

$$
\begin{array}{rl}
2 \sum_{\mathbf{p}} & f\left(\varepsilon_{\mathbf{p}}\right) \operatorname{Re}\left[a_{2}(y+i \delta, \mathbf{p})\right] \operatorname{Re}\left[b_{2}\left(x+i \boldsymbol{\epsilon}^{\prime}, \mathbf{p}\right)\right] \\
= & (1 / 4)\left\{h_{7}\left(x+i \epsilon^{\prime}, \omega+i \epsilon\right)+h_{7}\left(x-i \epsilon^{\prime}, \omega+i \epsilon\right)\right. \\
& +h_{7}\left(x+i \epsilon^{\prime}, \omega-i \epsilon\right)+h_{7}\left(x-i \epsilon^{\prime}, \omega-i \epsilon\right) \\
& +h_{8}(y+i \delta, \omega+i \boldsymbol{\epsilon})+h_{8}(y-i \delta, \omega+i \boldsymbol{\epsilon}) \\
& \left.+h_{8}(y+i \delta, \omega-i \boldsymbol{\epsilon})+h_{8}(y-i \delta, \omega-i \boldsymbol{\epsilon})\right\},
\end{array}
$$

$$
\begin{array}{rl}
2 \sum_{\mathbf{p}} & f\left(\varepsilon_{\mathbf{p}}\right) \operatorname{Im}\left[a_{1}(y+i \delta, \mathbf{p})\right] \operatorname{Re}\left[b_{1}\left(x+i \boldsymbol{\epsilon}^{\prime}, \mathbf{p}\right)\right] \\
& =H_{4}(\mathbf{q}, y ; \mathbf{k}, \omega),
\end{array}
$$

$$
\begin{array}{rl}
2 \sum_{\mathbf{p}} & f\left(\varepsilon_{\mathbf{p}}\right) \operatorname{Im}\left[a_{2}(y+i \delta, \mathbf{p})\right] \operatorname{Re}\left[b_{2}\left(x+i \boldsymbol{\epsilon}^{\prime}, \mathbf{p}\right)\right] \\
& =H_{8}(\mathbf{q}, y ; \mathbf{k}, \omega),
\end{array}
$$

$$
\begin{array}{rl}
2 \sum_{\mathbf{p}} & f\left(\varepsilon_{\mathbf{p}}\right) \operatorname{Im}\left[a_{1}(y+i \delta, \mathbf{p})\right] \operatorname{Im}\left[b_{1}\left(x+i \epsilon^{\prime}, \mathbf{p}\right)\right] \\
= & G_{4}(\mathbf{q}, y ; \mathbf{k}, \omega),
\end{array}
$$

$$
\begin{array}{rl}
2 \sum_{\mathbf{p}} & f\left(\varepsilon_{\mathbf{p}}\right) \operatorname{Im}\left[a_{2}(y+i \delta, \mathbf{p})\right] \operatorname{Im}\left[b_{2}\left(x+i \epsilon^{\prime}, \mathbf{p}\right)\right] \\
& =G_{8}(\mathbf{q}, y ; \mathbf{k}, \omega) .
\end{array}
$$

In conclusion, the entire set of $\mathbf{p}$ and $\mathbf{p}^{\prime}$ integrals appearing in $\operatorname{Im} \pi_{A B}(k, \omega)$ and $\operatorname{Im} \pi_{C}(k, \omega)$ is related to the eight functions $h_{i}$ and can be represented in terms of elementary functions.

\section{5. $q$ integration limits}

Before the numerical integration can be performed we must determine the integration limits. While the integration over the internal frequency $x$ extends from 0 to $\omega$, the $\mathbf{q}$ integration limits can be determined precisely (for a given $\omega$ and $x$ ) in the following way. As is clear from Eq. (A3), each term in $\operatorname{Im}\left[\pi_{A B}(k, \omega)+\pi_{C}(k, \omega)\right]$ contains a product of two imaginary parts of complex functions, one depending on $x$ and the other on $y=\omega-x$. These are $\operatorname{Im}\left[-1 / \epsilon_{L}(q, x)\right]$, $\operatorname{Im}\left[-1 / \epsilon_{L}(|\mathbf{q}+\mathbf{k}|, y)\right]$ or imaginary parts of one of the $h_{i}$ defined above. Apart from the plasmon poles, which are determined from $\epsilon_{L}(q, x)=0$, and $\epsilon_{L}(|\mathbf{q}+\mathbf{k}|, y)=0$, the loss functions are nonzero inside the particle-hole pair excitation spectrum, i.e., (in our units) for

$$
-1+\sqrt{1+x} \leqslant \sqrt{q_{\|}^{2}+q_{\perp}^{2}} \leqslant 1+\sqrt{1+x}
$$

and

$$
-1+\sqrt{1+y} \leqslant \sqrt{\left(q_{\|}+k\right)^{2}+q_{\perp}^{2}} \leqslant 1+\sqrt{1+y},
$$

respectively. To demonstrate this for the $h_{i}$ functions we consider, e.g.,

$$
\begin{aligned}
& \operatorname{Im} h_{1}(\mathbf{q}, y+i \delta ; \mathbf{k}, \omega) \\
& \quad \sim \int d^{3} p f\left(\varepsilon_{\mathbf{p}}\right) \delta\left(y+\varepsilon_{\mathbf{p}}-\varepsilon_{\mathbf{p}+\mathbf{k}+\mathbf{q}}\right) \cdots \\
& \quad \sim \int d p_{\|} \int d p_{\perp}^{2} \theta\left(1-p_{\|}^{2}-p_{\perp}^{2}\right) \delta\left(p_{0}-p_{\|}\right) \cdots \\
& \quad \sim \theta\left[\left(1-p_{0}\right)\left(1+p_{0}\right)\right] \cdots
\end{aligned}
$$

with $p_{0}=\left(y-|\mathbf{k}+\mathbf{q}|^{2}\right) /(2|\mathbf{k}+\mathbf{q}|)$. This means that $\operatorname{Im} h_{1}$ $\neq 0$ provided $-1+\sqrt{1+y} \leqslant \sqrt{\left(q_{\|}+k\right)^{2}+q_{\perp}^{2}} \leqslant 1+\sqrt{1+y}$ as a consequence of the $\Theta$ function and the $\delta$ function. Analogous manipulations can be performed for the other $\operatorname{Im} h_{i}$ to obtain the $\mathbf{q}$ integration limits. The actual integration limits follow from the cross section of the two $\mathbf{q}$ integration regimes related to the product of two imaginary parts.

The integrals with the above integration limits yield the contribution to $\operatorname{Im} \epsilon_{A B C}$ arising from ph-ph excitations. In addition, the poles from $\epsilon_{L}(q, x)$ and $\epsilon_{L}(|\mathbf{q}+\mathbf{k}|, y)$ yield contributions due to ph-pl excitation and pl-pl excitations. 
For the contribution arising from pl-pl excitations the $\mathbf{q}$ integration limits are defined by the section of the two semicircles:

$$
\begin{gathered}
0 \leqslant \sqrt{q_{\perp}^{2}+q_{\|}^{2}} \leqslant q_{c}, \\
0 \leqslant \sqrt{q_{\perp}^{2}+\left(q_{\|}+k\right)^{2}} \leqslant q_{c}
\end{gathered}
$$

This section is empty for $k>2 q_{c}$, i.e., pl-pl excitations are possible for $k<2 q_{c}$.

The q-integration limits for the contribution arising from ph-pl excitations are defined by the section of one of the conditions related to ph-ph excitations and one related to the pl-pl excitations.
*Present address: Chaire d'Electromagnétisme et de Télécommunications, Faculté Polytechnique de Mons, B 7000 Mons, Belgium.

${ }^{1}$ See, e.g., C. A. Ullrich, U. J. Gossmann, and E. K. Gross, Phys. Rev. Lett. 74, 872 (1995); G. Vignale, ibid. 74, 3233 (1995); G. Vignale and W. Kohn, ibid. 77, 2037 (1996); G. Vignale, C. A. Ullrich, and S. Conti, ibid. 79, 4878 (1997); P. Hessler, J. Park, and K. Burke, ibid. 82, 378 (1999).

${ }^{2}$ See, for example, S. Ichimaru, Rev. Mod. Phys. 54, 1017 (1982); N. Iwamoto, E. Krotscheck, and D. Pines, Phys. Rev. B 29, 3936 (1984); B. M. Farid, V. Heine, G. E. Engel, and I. J. Robertson, ibid. 45, 11602 (1993).

${ }^{3}$ J. Hubbard, Proc. R. Soc. London, Ser. A 243, 336 (1957).

${ }^{4}$ A. A. Kugler, J. Stat. Phys. 12, 35 (1975).

${ }^{5}$ A. Zangwill and P. Soven, Phys. Rev. A 21, 1561 (1980).

${ }^{6}$ E. K. U. Gross and W. Kohn, Phys. Rev. Lett. 55, 2850 (1985); 57, 923(E) (1986); N. Iwamoto and E. K. U. Gross, Phys. Rev. B 35, 3003 (1987).

${ }^{7}$ A. J. Glick and W. F. Long, Phys. Rev. B 4, 3455 (1971).

${ }^{8}$ H. M. Böhm, S. Conti, and M. P. Tosi, J. Phys.: Condens. Matter 8, 781 (1996).

${ }^{9}$ M. Hasegawa and M. Watabe, J. Phys. Soc. Jpn. 27, 1393 (1969).

${ }^{10}$ D. F. DuBois and M. G. Kivelson, Phys. Rev. 186, 409 (1969).

${ }^{11}$ C. F. Richardson and N. W. Ashcroft, Phys. Rev. B 50, 8170 (1994)

${ }^{12}$ V. I. Perel' and G. Éliashberg, Zh. Éksp. Teor. Fiz. 41, 886 (1961) [Sov. Phys. JETP 14, 633 (1962)].
${ }^{13}$ A. Ron and N. Tzoar, Phys. Rev. 131, 12 (1963).

${ }^{14}$ D. J. W. Geldart and S. H. Vosko, Can. J. Phys. 44, 2137 (1966).

${ }^{15}$ D. J. W. Geldart and R. Taylor, Can. J. Phys. 48, 167 (1970).

${ }^{16}$ D. Langreth and S. H. Vosko, Phys. Rev. Lett. 59, 497 (1987).

${ }^{17}$ A. J. Glick, Phys. Rev. 129, 1399 (1963); A. Holas, P. K. Aravind, and K. S. Singwi, Phys. Rev. B 20, 4912 (1979).

${ }^{18}$ J. Lindhard, K. Dan. Vidensk. Selsk. Mat. Fys. Medd. 28, 8 (1954).

${ }^{19}$ M. E. Bachlechner, A. Holas, H. M. Böhm, and A. Schinner, Phys. Rev. B 54, 2350 (1996).

${ }^{20}$ K. Sturm, Adv. Phys. 31, 1 (1982).

${ }^{21}$ S. L. Adler, Phys. Rev. 126, 413 (1962); N. Wiser, ibid. 129, 62 (1963).

${ }^{22}$ P. J. Zacharias, J. Phys. F: Met. Phys. 5, 645 (1975).

${ }^{23}$ W. Schülke, H. Schulte-Schrepping, and J. R. Schmitz, Phys. Rev. B 47, 12426 (1993).

${ }^{24}$ P. M. Platzman, E. D. Isaacs, H. Williams, P. Zschack, and G. E. Ice, Phys. Rev. B 46, 12943 (1992).

${ }^{25}$ J. J. Hopfield, Phys. Rev. 139, A419 (1965).

${ }^{26}$ N. V. Smith, Phys. Rev. Lett. 21, 96 (1968); Phys. Rev. 183, 634 (1969); Phys. Rev. B 2, 2840 (1970).

${ }^{27}$ B. I. Bennett and S. H. Vosko, Phys. Rev. B 6, 2116 (1972).

${ }^{28}$ D. J. Stevenson, Phys. Rev. B 7, 2348 (1973).

${ }^{29}$ S. Moroni, D. Ceperley, and G. Senatore, Phys. Rev. Lett. 75, 689 (1995). 